\title{
Elastocapillarity in nanopores: Sorption strain from the actions of surface tension and surface stress
}

\author{
Gennady Y. Gor, ${ }^{1, *}$ Patrick Huber, ${ }^{2, \dagger}$ and Jörg Weissmüller ${ }^{2,3, \ddagger}$ \\ ${ }^{1}$ Otto H. York Department of Chemical and Materials Engineering, New Jersey Institute of Technology, University Heights, \\ Newark, New Jersey 07102, USA \\ ${ }^{2}$ Institute of Materials Physics and Technology, Hamburg University of Technology, Hamburg, Germany \\ ${ }^{3}$ Institute of Materials Research, Materials Mechanics, Helmholtz-Zentrum Geesthacht, Geesthacht, Germany
}

(Received 7 April 2018; published 31 August 2018)

\begin{abstract}
Adsorption-induced deformation of porous materials is the generation of strains in a solid due to its interaction with adsorbing fluids. The theoretical description of adsorption-induced deformation often relies on the so-called solvation pressure, the normal component of a pressure tensor in the liquid adsorbed in the pore. Recent measurements of adsorption-induced strains in two dimensions require a description that allows for the deformation to be anisotropic. Here, we present such a description. We refrain from using the solvation pressure concept and instead base the discussion on a phenomenological description of coupled mechanics and adsorption that has well-established links to continuum mechanics. We find that our approach captures all relevant features of anisotropic sorption strain; the approach thus provides a useful alternative to the solvation pressure concept. We derive analytical expressions for the stress-strain relations in a model porous material with an array of parallel channel-like pores of high aspect ratio (length/width). These relations include separate terms from the liquid pressure, from the surface stress at the liquid-solid interface, and from a spreading tension at the solid-liquid-vapor triple line. Surface stress and liquid pressure contribute to the strains along and normal to the pore axis in a qualitatively different manner. The underlying discussion of capillary forces sheds light on the variation of the surface stress during adsorption and capillary condensation.
\end{abstract}

DOI: 10.1103/PhysRevMaterials.2.086002

\section{INTRODUCTION}

Stress and strain are generated in porous solids when matter adsorbs on the pore surfaces [1]. The solid may be in contact with gas, where the vapor pressure of the adsorbate controls the adsorption process. Alternatively, the solid may be in contact with a solution containing the adsorbing species in the form of dissolved molecules or ions, in which case a chemical or electric potential controls the adsorption. In simple scenarios the fluid (vapor or solution) extends uniformly from the outside of the porous solid into the pore space. The solid is then loaded by the combination of the hydrostatic pressure of the fluid and by capillary forces that act along the pore walls. More complex scenarios are found during capillary condensation of undersaturated vapors [2] and imbibition of wetting liquids [3-9] as well as forced intrusion of nonwetting liquids $[10,11]$. Here, menisci form between the outer fluid and the liquid in pore space. This leads to a mechanical loading scenario in which part of the solid surface is loaded by the pressure in the liquid, while simultaneously capillary traction forces act at the liquid-solid-vapor triple lines and the liquid-solid

\footnotetext{
*Corresponding author: gor@njit.edu

†patrick.huber@tuhh.de

†weissmueller@tuhh.de
}

Published by the American Physical Society under the terms of the Creative Commons Attribution 4.0 International license. Further distribution of this work must maintain attribution to the author(s) and the published article's title, journal citation, and DOI. interfaces experience surface stress. The coupling of liquid capillarity and solid elasticity is here similar or even more complex as encountered in planar wetting geometries [12-16] or for droplet spreading on flexible fibers and wires $[13,17,18]$ upon liquid-vapor coexistence.

The strains in each of the above situations have practical implications, for instance in the context of sensing and actuation. Such examples include a porous polymer, responding with significant strain when water or acetone vapors adsorb on its surface [19]; an actuating device made of a microcantilever with a layer of mesoporous silica deposited on one of its sides, strain induced by adsorption of water vapor on the silica walls causes bending of the cantilever [20,21]. Another actuating device was synthesized in a form of an artificial pine cone, made of porous silica, which is able to mimic the behavior of its natural prototype: adsorption-induced deformation makes the ovuliferous scales of the cone move with the change of humidity [22].

Nanoporous materials saturated with gas or electrolyte provide a striking example where the fluid is uniform and menisci absent. Nanoporous gold permeated by gas can act as an actuator driven by changes in the partial pressure of adsorbing species in the gas [23]. Sorption from gas, and specifically the sorption-induced expansion of coal, is also significant for geology and the exploitation of fossil fuels [24-26]. Nanoporous carbons [27-29] and metals [30-33] permeated by electrolyte react to electric signals by reversible strain. The porous metal also exhibits the inverse effect, namely, the generation of electric signals in response to external load [34]. Thus, the coupling between mechanics and (electro)chemistry 
at pore surfaces may even induce piezoelectricity classically a prerogative of ceramics in metals [35].

First measurements of adsorption-induced strains were reported by Meehan [36] and Bangham and co-workers [37] for various gases on charcoal. Experiments on mesoporous Vycor glass [38,39] and zeolites [40,41] followed. In recent years, adsorption-induced deformation was measured for many novel synthetic materials: microporous and mesoporous carbons [42-44], mesoporous silica [45-48], mesoporous silicon [49,50], metal-organic frameworks [51,52]. Electrosorption phenomena in porous solids were first reported by Beck [53]. Later work used cantilever bending studies and planar surfaces to precisely quantify the underlying electrocapillary coupling effects [54-58], which are applied in modern nanoporous metal actuation schemes [59].

Theoretical studies of adsorption-induced deformation have presented various thermodynamic models based on macroscopic $[60,61]$ and microscopic $[62,63]$ descriptions as well as mechanical models $[50,64,65]$. Atomistic simulation of the deformation of porous solids by capillary forces provides a test bed for such theories $[66,67]$.

Although using different experimental methods, typically experimental works report a single measure for the strain. The same applies to most of the theoretical considerations. Deformation of disordered materials can often be considered isotropic. Yet, as the deformation varies the volumes of the solid and of the pore space independently, a single scalar strain value may not appropriately describe the deformation. This has been demonstrated by experiments on nanoporous metals [68] and by atomistic simulation $[66,69]$. Ordered materials with microstructural anisotropy may require an even more complex description. In 2015 two experiments reported adsorptioninduced strains for porous materials with anisotropic structure performed in two different directions [70,71]. Balzer et al. [70] reported the measurements of strains in hierarchical silica monoliths, composed of long struts with channel-like mesopores oriented along the struts. Using in situ small-angle $\mathrm{x}$-ray scattering (SAXS) they measured the strain of the pores normal to the pore walls, and using dilatometry they measured the strain of the monolithic sample, related to the deformation both normal to and along the pore walls. Grosman et al. [71,72] studied the strains in mesoporous silicon membranes. Similar to the silica samples from Balzer et al., this material has an array of channel-like parallel pores. Strains normal to the pore walls and along the pore walls were measured by dilatometry. Both of these works showed a difference between the strains normal to the pore walls and along the pore walls. This stimulated the development of a three-dimensional (3D) model of adsorption-induced deformation for such systems, which is the focus of the current paper.

Theory can approach adsorption-induced stresses at the pore walls in two alternative ways. The first one is based on the so-called solvation pressure. In the field of solid mechanics, pressure is a scalar quantity, defined as $-\frac{1}{3}$ of the trace of a stress tensor. In that field, bulk fluids at rest are uniform and are understood to be distinguished by their hydrostatic stress state. Yet, molecular fluids can be inhomogeneous in the regions adjacent to a solid surface, and this entails nonhydrostatic stress states. Their discussion involves what is often referred to as a pressure tensor $[73,74]$, and the solvation pressure is defined as a normal component of this quantity [75]. Thus, the adsorptioninduced deformation phenomenon is typically described in terms of one scalar pressure variable. The second approach is based on the surface stress at the solid surface. This approach considers the fluid as uniform and hydrostatic, while accounting for the interactions in the interfacial regions in terms of a 2D surface which exhibits tangential stress. On curved surfaces, the tangential stress entails a normal stress component. One of us has shown recently that the two approaches to local stress states can be consistent in the case of non-site-specific adsorption [76]. Yet, there is ample experimental evidence for the relevance of site-specific adsorption and of associated changes in the surface stress. The two approaches, solvation pressure versus surface stress, appear to remain separate and possibly even mutually exclusive when it comes to those more general adsorption scenarios. Furthermore, their consequences for adsorption-induced strains during capillary condensation have not been compared so far.

Here, we explore in how far the deformation of porous solids in the various stages of capillary condensation can be described by the concept of surface stress, and we compare the results to predictions based on the concept of solvation pressure. We explicitly consider both adsorption scenarios, site-specific and non-site-specific. Closed-form expressions are obtained for the stress-strain relations in a model porous material with an array of parallel channel-like pores in the limit of high aspect ratio. The results include separate terms from the fluid pressure and from the surface stress at the fluid-solid interface and the liquid-solid-vapor triple lines after capillary condensation. These terms contribute to the strains along the pore axis and normal to the pore axis in a qualitatively different fashion. Based on the stress-strain relations, we calculate the pore-load moduli corresponding to the strains normal to and along the pore walls. The normal pore-load modulus is close to that of a 2D model [50], while the parallel pore-load modulus, which is beyond that of the 2D model, has a noticeably different value. Our results establish the description in terms of surface stress as a valid approach to analyzing the deformation of porous solids during capillary condensation.

\section{PORE-FILLING REGIMES}

Our discussion starts out with the brief introduction of the three regimes of the pore filling and capillary condensation process, as schematically illustrated in Fig. 1. We consider a solid matrix, containing cylindrical pores, in contact with the vapor (V) of the adsorbate. We consider mesoporous materials, i.e., the pore sizes in our discussion are in the range above $2 \mathrm{~nm}$, so that the macroscopic concepts of liquid films and capillary condensation can be used. The pressure $p$ of the vapor is controlled and provides the experimental control variable that governs the process of pore filling. This pressure controls the chemical potential $\mu$ of the adsorbate and can be parametrized by $\mu$. We consider an initial state (Fig. 1I) with $p=0$, so that the pore walls are clean, and we investigate the evolution of the system as $p$ is gradually increased.

Increasing $p$ will initially result in the adsorption of submonolayer quantities on the pore walls (Fig. 1II). This is followed by the formation of a thicker layer that may comprise few molecular monolayers (Fig. 1III). Up to this point, the net 


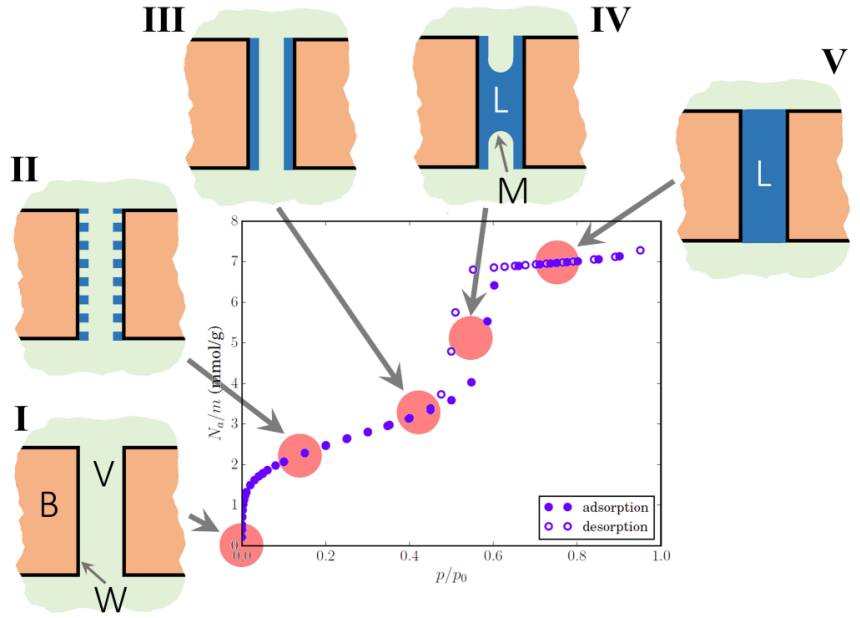

FIG. 1. Schematic illustration of pore-filling regimes. Background image: adsorption isotherm of adsorbed/condensed amount $N$ of matter versus reduced pressure $p / p_{0}$. Schematics show pore filling regimes, starting with the clean surfaces (I) and progressing through adsorption of a partial (II) and then complete (III) monolayer, capillary condensation (IV), and (V) approach to saturation. Labels denote the phases and interfaces: $\mathrm{B}$, solid bulk; V, vapor; L, liquid; $W$, pore wall; $M$, meniscus. The data are taken from Ref. [77] for nitrogen adsorption on mesoporous silica.

quantity of adsorbed matter scales with the area of pore surface. Further increase of $p$ triggers capillary condensation, that is, the onset of filling of the pore with liquid (Fig. 1IV). Once this process is complete, the net amount of absorbed matter scales with the volume of the pores. The condensate is separated from the vapor by a concave meniscus. As the pressure is further increased, the meniscus approaches a planar configuration, while the pores approach saturation (Fig. 1V). These processes lead to an adsorption isotherm with two distinct regions, separated by a capillary condensation point. Typically the capillary evaporation during the desorption process takes place at the lower vapor pressure than the capillary condensation. The sorption isotherm then has a hysteresis loop, as exemplified in Fig. 1. Detailed discussion of the hysteresis can be found in the recent reviews [78-80].

\section{INTERFACES AND CAPILLARY TERMS}

The relevant interfaces in our problem are first, the meniscus (M) that separates the liquid (L) in the pores from the vapor $(\mathrm{V})$, and second the pore walls (W), that is, the interface between the solid bulk (B) and the vapor (in the adsorption regime) or the liquid (in the capillary condensation and approach to saturation regimes). Each of these interfaces is associated with a surface tension (i.e., an excess energy per area of surface) $\gamma$ and we designate the three surface tensions by $\gamma^{\mathrm{LV}}, \gamma^{\mathrm{BV}}, \gamma^{\mathrm{BL}}$.

In fluids, the specific excess free energy or surface tension is inherently linked to the pressure via the Young-Laplace equation: across a fluid-fluid interface with a mean curvature $\kappa$, the pressure jumps by $2 \kappa \gamma$. It is important to note that this equation applies to the meniscus but does not apply to the interface between the solid and the fluid. Here, the effect of the interface on the mechanics is governed by the surface stress $\mathbb{S}$.
For an in-depth discussion of the distinction between $\gamma$ and $\mathbb{S}$, the reader is referred to Ref. [81]. In brief, the surface tension quantifies the work which is done against the surface when atoms are added to it at constant atomic structure; this deformation mode is characteristic of the surface of a liquid. By contrast, the surface stress refers to the work done when elastically deforming the surface, keeping the number of atoms constant but changing their spacing. This characterizes the elastic deformation of the surface of a solid. The surface stress has the same units and similar numerical magnitude as the surface tension, yet its mathematical representation differs: whereas the surface tension may be described by a scalar, the surface stress describes a directional stress in the tangent plane of the surface and thus requires a representation as a second-rank tensor. The surface-stress state may be anisotropic in the plane. Furthermore, whereas stability criteria constrain the surface tension to positive values, the entries of the surfacestress tensor may be of either sign.

For the problem at hand, $\gamma$ is the relevant parameter when the meniscus is deformed since this process changes the number of atoms in the liquid's surface. By contrast, elastic deformation of the solid matrix changes the interatomic spacing in the pore walls, thereby working against $\mathbb{S}$.

\section{CONSTITUTIVE STATEMENTS}

\section{A. Free energy}

This section briefly introduces a phenomenological thermodynamics description of surface mechanics and of its coupling to adsorption. More details can be found in Ref. [34]. We start out with an expression for the net free energy of the porous solid. We take the solid as an elastic substrate and the liquid as an incompressible fluid. The energetics of the bulk phases are represented by free-energy densities (per volume $V$ ), $\Psi^{\mathrm{B}}$ for the solid and $\Psi^{\mathrm{L}}$ for the liquid, and by the superficial free-energy densities $\psi$ (per area $A$ of surface). The free-energy densities are defined so that the net free energy $\mathfrak{F}$ obeys

$$
\mathfrak{F}=\int_{\mathrm{B}} \Psi^{\mathrm{B}} d V+\int_{\mathrm{W}} \psi^{\mathrm{W}} d A+\hat{\Psi}^{\mathrm{L}} \hat{V}^{\mathrm{L}}+\hat{\psi}^{\mathrm{M}} \hat{A}^{\mathrm{M}},
$$

where the subscripts denote the respective regions. The equation is based on the notion that the fluid is uniform, whereas the solid will in general contain gradients in stress and strain and it may be bordered by interfaces with different fluids.

Discussion of the mechanics of the solid surface is considerably simplified by the use of "Lagrangian" coordinates, where $V$ and $A$ are measured not in the actual, strained state of the solid ("Eulerian" coordinates), but in a strain-free reference configuration $[81,82]$. The alternative use of Lagrangian or Eulerian coordinates is routine in solid mechanics in three dimensions, where it underlies the distinction between Cauchy and second Piola-Kirchhoff stress measures (see for instance the textbook by Bonet and Wood [83]). The generalization of this concept to surfaces, which underlies a standard approach to surface mechanics $[84,85]$, is adopted here. In particular, therefore, the densities $\Psi^{\mathrm{B}}$ and $\psi^{\mathrm{W}}$ are defined as energy in the actual state, per volume or per area of surface in the undeformed state. The "hatted" quantities in Eq. (1), $\hat{\psi}^{\mathrm{M}}$ and $\hat{A}^{\mathrm{M}}$, refer entirely to the fluid. Here, the concepts of strain and 
of reference configuration are not appropriate, and densities are referred to volume $\hat{V}$ and area $\hat{A}$ in the actual configuration.

Quite generally, throughout this work, the hat denotes quantities measured in actual (laboratory) coordinates. This convention is also relevant when defining the superficial excess $\Gamma$ of the adsorbate. $\Gamma$ is defined as the excess, per referential area, of the actual amount of adsorbate in the system over the amount in the same volume of pore if there was no surface. For instance, if the pore wall is completely wetted, then the net amount of adsorbate in the system is

$$
N=\hat{\rho}_{0} \hat{V}^{\mathrm{L}}+\Gamma A^{\mathrm{BL}} .
$$

At this point, we introduce the parameter $L$, which translates between areas of surface measured in referential and laboratory coordinates, respectively (see Ref. [34] for details):

$$
L=\hat{A} / A=1+e .
$$

The area-strain parameter $e$ denotes the relative variation in surface area (in laboratory coordinates) by elastic strain of the underlying bulk. The superficial densities transform as $\psi=$ $L \hat{\psi}, \Gamma=L \hat{\Gamma}$.

We restrict attention to isothermal processes and take $\Psi^{\mathrm{B}}=$ $\Psi^{\mathrm{B}}(\mathbf{E})$ as the constitutive assumption for the (Helmholtz-type) free-energy density function of the solid. $\mathbf{E}$ denotes the strain tensor. The fundamental equation for $\Psi$ is then

$$
d \Psi^{\mathrm{B}}=\mathbf{S}: d \mathbf{E}
$$

with $\mathbf{S}$ the stress tensor [86].

As the liquid is assumed incompressible, we can take (for isothermal processes)

$$
\begin{aligned}
& \hat{\Psi}^{\mathrm{L}}=\hat{\Psi}_{0}^{\mathrm{L}}=\mathrm{constant} \\
& \hat{\psi}^{\mathrm{M}}=\hat{\psi}_{0}^{\mathrm{M}}=\mathrm{constant} .
\end{aligned}
$$

Contrary to the liquid-vapor interface, the energy of the surfaces of the solid can vary as a function of the strain in the substrate. Furthermore, the $\psi$ will depend on $\Gamma$. The considerations so far are consistent with constitutive and fundamental equations for the surfaces of the solid in the form [34]

$$
\begin{gathered}
\psi^{\mathrm{W}}=\psi^{\mathrm{W}}(\Gamma, \mathbb{E}), \\
d \psi^{\mathrm{W}}=\mu d \Gamma+\mathbb{S}: d \mathbb{E},
\end{gathered}
$$

with $\mu$ the chemical potential of the adsorbate, $\mathbb{S}$ the surface stress, and $\mathbb{E}$ the tangential strain tensor, a projection of the strain $\mathbf{E}$ in the regions of bulk near the interface into the local tangent plane.

Henceforth, we shall simplify the discussion by assuming all surfaces to be isotropic in the plane. Then, $\mathbb{S}=f \mathbb{P}$ with $f$ a scalar surface-stress parameter and $\mathbb{P}$ a unit tensor in the local tangent plane. The constitutive and fundamental equations then simplify to

$$
\begin{gathered}
\psi^{\mathrm{W}}=\psi^{\mathrm{W}}(\Gamma, e), \\
d \psi^{\mathrm{W}}=\mu d \Gamma+f d e,
\end{gathered}
$$

respectively. Here $f=\frac{1}{2} \operatorname{Tr} \mathbb{S}$ is a scalar surface-stress variable.
The state functions for the free-energy density of the solid surfaces $\psi(\Gamma, e)$ are related to the surface tensions $\gamma(\mu, e)$ by Legendre transforms such as

$$
\begin{gathered}
\gamma(\mu, e)=\psi(\Gamma, e)-\mu \Gamma, \\
d \gamma=-\Gamma d \mu+f d e .
\end{gathered}
$$

Equation (12) is a generalized Gibbs adsorption equation that applies to solid surfaces (and is here written for isothermal processes). For the fluid-fluid interface at the meniscus we have, consistent with our assumptions, simply $\gamma^{\mathrm{M}}=\psi^{\mathrm{M}}=$ constant.

A consequence of the above considerations is that elastic deformation at constant referential area (constant number of surface atoms) changes the net surface free energy of a uniform section of surface by $A d \psi=A f d e$. By contrast, if the referential area of the section of surface is changed by varying its number of surface atoms at constant strain, then the change in free energy is $\psi d A$ or $\gamma d A$, depending on whether $\Gamma$ or $\mu$ are held constant.

The distinction between Eq. (12) and the Shuttleworth equation [87], $f=\partial \gamma / \partial e$ versus $f=\hat{\gamma}+\partial \hat{\gamma} / \partial e$, is discussed in detail in Ref. [81]. The two equations are equivalent and they describe the same physics. Their formal dissimilarity is simply the consequence of the different reference frames, Lagrangian versus Eulerian. Yet, the discussion of surface stress, for instance by analysis of thermodynamic derivatives, is drastically simplified when working with Eq. (12). In a field that is riddled with dubious statements [81], the superior conceptional clarity of that latter formulation must be considered a decisive asset, which motivates our preference for Lagrangian coordinates.

\section{B. Adsorption: Site specific versus incommensurate}

Adsorption processes may, in a simplified picture, be distinguished as either site specific or nonspecific (incommensurate). The distinction is of relevance since we shall argue that the coupling between elastic strain of the solid surface and the superficial excess or chemical potential of the adsorbate differs fundamentally between the two modes of adsorption.

Atoms or molecules adsorbing at a surface typically tend to attach to specific sites, and solid surfaces may be characterized by their density $\Gamma_{\text {site }}$ of sites per area. When the interaction of the adsorbate with the surface is sufficiently attractive, the specific adsorption may dominate in the early stages of adsorption [88,89]. For metal surfaces, the adsorption of a second metal, of small ions such as protons, inorganic anions, or of organic molecules that are functionalized by thiol or amine groups provide examples [90,91].

As the coverage $\Gamma / \Gamma_{\text {site }}$ approaches unity, the available sites for specific adsorption become exhausted. This is one instance where a commensurate-incommensurate transition may occur [88]. The incommensurate layer is distinguished by its lack of registry with the solid: the amount of excess in this layer depends on the adsorbate chemical potential and on the solution chemistry of a possible solvent, but will (ideally) not depend on details of the crystallography of the surface.

The underpotential deposition of $\mathrm{Bi}$ on $\mathrm{Au}$ from electrolyte $[92,93]$ and the adsorption of $\mathrm{Kr}[88,94]$ on planar graphite are prominent examples of such commensurate-incommensurate 
transitions. More generally, electrode surfaces in interfacial electrochemistry exhibit (site-) specifically adsorbed ions in their inner Helmholtz layer and a diffuse (and incommensurate) cloud of solvated ions in the outer Helmholtz layer [95]. Amorphous surfaces naturally favor short-range ordered interfacial layers. Examples are the pore-wall adsorbed amorphous monolayers of simple van der Waals liquids, such as argon, oxygen, and nitrogen [2,96-99], water [100], and linear hydrocarbons [101] in nanoporous amorphous silica as well as a variety of polymeric systems in nanoporous anodic aluminum oxide substrates [102]. Liquids, most prominently water, can also form ordered interfacial layers near the interface with a crystal surface [103]. The first molecular layer of a liquid may be commensurate with the solid [88,104], while the absence of shear stress in the bulk liquid forbids a commensurate structure in the more bulklike regions of a liquid away from the interface. Since we are motivated by an example of adsorption on amorphous silica surface, we focus on the incommensurate phases.

\section{Equations of state: Solid-vapor surface tension}

When the pressure in the vapor and, along with it, the chemical potential of the adsorbate are increased, the surface tensions, surface free-energy densities, and surface stresses of the interfaces of the solid with liquid and vapor vary. We shall now discuss these variations, starting out with the surface tension.

The change of the surface tension $\Delta \gamma$ of the solid-vapor interface due to adsorption may be obtained by integrating the Gibbs adsorption equation (12) [105]:

$$
\Delta \gamma^{\mathrm{BV}}=-\int_{-\infty}^{\mu} \Gamma(\tilde{\mu}) d \tilde{\mu} .
$$

For extremely small pores, corrections due to curvature may apply. Yet, as it was shown in Ref. [61], these corrections may be ignored except for a small vicinity of the capillary condensation point.

Explicitly evaluating the integral in Eq. (13) requires an adsorption isotherm in the form $\Gamma=\Gamma(\mu)$. Following Ref. [76] we here focus on the Brunauer-Emmett-Teller (BET) isotherm [106], which is one of the most widely used theories for multilayer adsorption of fluids on solid surfaces. The BET adsorption isotherm is given by

$$
\Gamma\left(p / p_{0}\right)=\Gamma_{\mathrm{m}} \frac{C p / p_{0}}{\left(1-p / p_{0}\right)\left[1+(C-1) p / p_{0}\right]},
$$

where $\Gamma$ is the amount of fluid adsorbed per unit area of the solid adsorbent, $p$ is the adsorbate vapor pressure, $p_{0}$ is the pressure of the saturated vapor, and $\Gamma_{\mathrm{m}}$ and $C$ are the only two parameters of the model: the capacity of the monolayer and the BET constant. The vapor pressures $p$ and $p_{0}$ relate to the chemical potential as

$$
\mu=\mu_{0}+R_{\mathrm{g}} T \ln \frac{p}{p_{0}},
$$

with $\mu_{0}$ the chemical potential of the saturated vapor and $R_{\mathrm{g}}$ the gas constant.



FIG. 2. Experimental adsorption-induced strain $\epsilon$ versus the reduced pressure $p / p_{0}$. Measurement by in situ dilatometry for hierarchical mesoporous silica during nitrogen adsorption [77].

With Eq. (14), the Gibbs adsorption equation [Eq. (13)] is here conveniently integrated. Using Eq. (15), we obtain

$$
\Delta \gamma^{\mathrm{BV}}=-R_{\mathrm{g}} T \Gamma_{\mathrm{m}} \ln \left[\frac{1+(C-1) p / p_{0}}{1-p / p_{0}}\right] .
$$

This expression determines the change of the solid-vapor surface tension in the region prior to the capillary condensation.

\section{Equations of state: Solid-liquid surface tension}

As exemplified in Fig. 2, experimental sorption strain data exhibit discontinuity during capillary condensation and evaporation. Similar discontinuities have been reported for the strain isotherms on many mesoporous materials [1]. This observation must be related to discontinuities in surface tension, surface stress, and in the pressure in the pore space. Theory predictions for these discontinuities are shown below in Fig. 6(b). Discussing the magnitude of the strain discontinuities therefore requires, specifically, information on the surface tension of the solid-liquid interface. One might be tempted to integrate Eq. (13) up to $\mu=\mu_{0}$ and identify the result with the above-mentioned quantity. However, phenomenological thermodynamics provides no basis for this approach. This is obvious notably when one inspects the definition of the integration variable $\Gamma$. For the solid-vapor interface, $\Gamma$ is an excess of adsorbate over the clean solid surface adjacent to vacuum and, as such, always positive. For BET adsorption, $\Gamma$ at finite $p$ can amount to several molecular monolayers. By contrast, $\Gamma$ for the liquid-vapor interface is an excess of matter over the interface in contact with a perfectly homogeneous fluid. That excess, for instance representative of liquid layering near the interface, may be small or even negative. The discontinuity in $\Gamma$ illustrates that the integration in Eq. (13) cannot be carried continuously through the interfacial phase transformation between solid-vapor and solid-liquid.

Unlike the excess in matter, the local molecular structure in the immediate vicinity of the interface is expected to be quite similar for the thick adsorbate layer at $\mu<\mu_{0}$ and for the interface between the bulk fluid and the solid at $\mu=\mu_{0}$. 


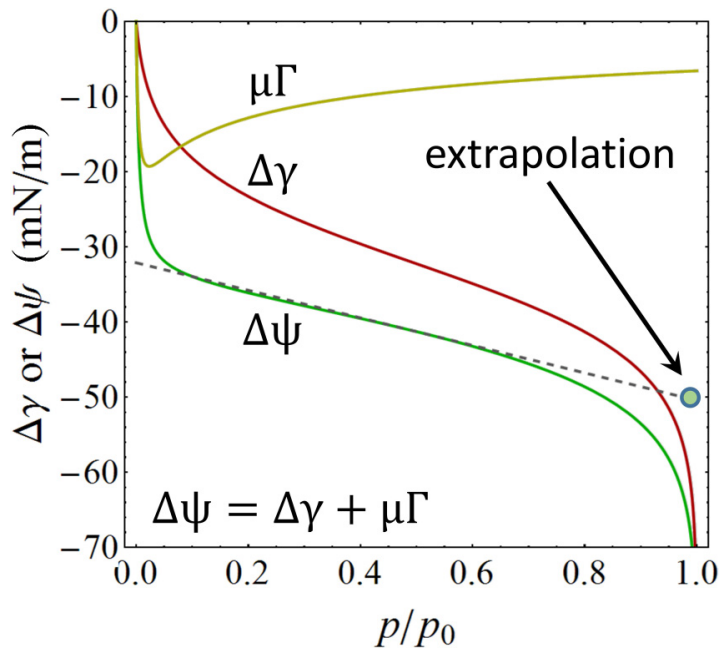

FIG. 3. Changes $\Delta \psi$ and $\Delta \gamma$ of the surface free-energy density and surface tension, respectively, for the BET adsorption isotherm. Also shown is the difference, namely the product $\mu \Gamma$ of $\mu$, the chemical potential, and $\Gamma$, the specific excess in adsorbate. Dashed line: linear extrapolation of $\Delta \psi$ based on the slope at $p / p_{0}=0.5$. Based on parameters for nitrogen adsorption on silica (see Sec. VI).

Since the free-energy density is governed by the local atomic configurations, we may then take the free-energy densities to be similar as well. This suggests that

$$
\left.\left.\psi^{\mathrm{BL}}\right|_{\mu=\mu_{0}} \approx \psi^{\mathrm{BV}}\right|_{\mu=\mu_{0}} .
$$

In view of Eqs. (11) and (13), we may therefore write for the surface tension

$$
\left.\left.\gamma^{\mathrm{BL}}\right|_{\mu=\mu_{0}} \approx \psi^{\mathrm{BV}}\right|_{\mu \rightarrow \mu_{0}}-\left.\mu_{0} \Gamma^{\mathrm{BL}}\right|_{\mu=\mu_{0}} .
$$

Capillary condensation implies solid-liquid interfaces at chemical potentials $\mu<\mu_{0}$; here,

$$
\left.\left.\gamma^{\mathrm{BL}}\right|_{\mu<\mu_{0}} \approx \psi^{\mathrm{BV}}\right|_{\mu \rightarrow \mu_{0}}-\left.\mu_{0} \Gamma^{\mathrm{BL}}\right|_{\mu=\mu_{0}}-\int_{\mu_{0}}^{\mu} \Gamma^{\mathrm{BL}}(\tilde{\mu}) d \tilde{\mu} .
$$

We now explore the above estimate for the example of the BET isotherm [Eq. (14)]. That isotherm is valid only at low $p: \Gamma$ diverges at $p / p_{0} \rightarrow 1$, and so does $\Delta \psi$, precluding an extrapolation in the sense of Eq. (18). Yet, the BET $\psi^{\mathrm{BV}}$ has an extended region of nearly linear variation with $p$ (Fig. 3), and we here extrapolate this region linearly from $p / p_{0}=0.5$ to $p / p_{0}=1.0$. Accounting for Eq. (16) and for $\psi=\gamma+\mu \Gamma$ we find for the extrapolated $\psi$ at $p=p_{0}$

$$
\left.\Delta \psi^{\mathrm{BV}}\right|_{\mu=\mu_{0}} \approx-R_{\mathrm{g}} T \Gamma_{\mathrm{m}}\left[\ln (C+1)+\frac{4 C(C+2) \ln 2}{(1+C)^{2}}\right] .
$$

Inasmuch as $\mu_{0}$ can, without lack of generality, be defined as the zero value of the chemical potential for the isothermal processes considered here, Eq. (20) also provides an estimate for (the incommensurate part of) $\gamma^{\mathrm{BL}}$ at $p=p_{0}$. With BET parameters for nitrogen adsorption on silica (see Sec. VI below), one then obtains $\left.\Delta \gamma^{\mathrm{BL}}\right|_{\mu=\mu_{0}}=-50.4 \mathrm{mN} / \mathrm{m}$. This agrees with the $-50 \mathrm{mN} / \mathrm{m}$ that result from the calculation of
$\Delta \gamma^{\mathrm{BL}}$ based on the integration of the exponential disjoining pressure isotherm [77]. Apparently, the extrapolation captures at least roughly the energetics of the solid-liquid interface.

\section{E. Equations of state: Surface stress}

Since the surface stress is not a thermodynamic potential, its variation with the state variables $\Gamma$ or $\mu$ is not restricted by an adsorption equation of similarly fundamental character as Eq. (12) for $\gamma$. Our discussion of equations of state for $f$ accounts for empirical observations and simple models.

Early stage. Experimental studies of surface-stress variation during specific adsorption of ions from electrolyte indicate a roughly linear correlation, which can be quantified by an electrocapillary coupling parameter $\varsigma$ that is characteristic of the combination of solid surface and adsorbing ion [107,108]. The coupling between surface stress and adsorbate coverage may be understood as a consequence of changes in the electron density in the bonding regions between the surface atoms and of out-of-plane relaxation in the outermost atomic layers of the solid [109]. Electrocapillary coupling coefficients for metal surfaces in contact with electrolyte are often quite similar to those with gas [110]. Thus, it appears natural to approximate the surface-stress variation in the early stages of pore filling at not too high coverage (for instance, up to one molecular monolayer) by a linear variation with coverage:

$$
f^{\mathrm{BV}}=f_{0}+\zeta \Gamma .
$$

Here, $f_{0}$ and $\zeta$ denote the surface stress of the clean solid surface and a coupling parameter for adsorption from vapor, respectively. If the surface-stress change per adsorbate were identical for adsorption from vapor and for electrosorption, then $\zeta$ was related to the electrocapillary coupling parameter $\varsigma$ by $\zeta=-z F \varsigma$ [110]. In other words, the typical experimental values of $\varsigma$, in the order of -1 to $-2 \mathrm{~V}$ for negatively charged (valency $z<0$ ) species [110,111], would translate into $\zeta=-1$ to $-2 \mathrm{eV} /$ atom. With typical site densities at solid surfaces in the order of 10 to 20 sites $/ \mathrm{nm}^{2}$, a full monolayer of adsorbate may then change the surface-stress magnitude by several $\mathrm{N} / \mathrm{m}$. Experiment indeed supports such large changes [23]. This effect is quite significant and may not, a priori, be neglected. Site-specific adsorption typically makes $f$ more negative $[93,107,108]$, but the opposite effect, increasing $f$ during adsorption, has also been reported [112,113].

Advanced stage. When the coverage exceeds one monolayer, the mechanism and magnitude of the chemomechanical coupling at the surface must be expected to change. As we have argued above, a not unrealistic scenario is that the second and higher layers of adsorbate are incommensurate with the surface. We now show that this scenario leads naturally to a variant of Bangham's law for the surface-stress variation, which generalizes the classic Bangham's law that has been discussed in the context of deformation of microporous materials [76].

We assume that the net specific excess in adsorbate $\Gamma$ can be decomposed into contributions from the two modes, site specific $\left(\Gamma_{\mathrm{S}}\right)$ and incommensurate $\left(\Gamma_{\mathrm{I}}\right)$. Site-specific adsorption is distinguished by an explicit dependence of the surface free energy on strain, which takes account of the fact that changing the bond lengths in the substrate will, in general, 
change adsorption enthalpies [113,114]. Thus, the contribution of this "inner" layer to the net surface free-energy density may be represented by the fundamental equation $\psi_{\mathrm{S}}=\psi_{\mathrm{S}}\left(\Gamma_{\mathrm{S}}, e\right)$. By contrast, the contribution of an incommensurate layer to the surface free energy will not explicitly depend on the state of strain of the surface. It is therefore natural to represent the energetics of this layer in laboratory coordinates and to assume that $\hat{\psi}_{\mathrm{I}}=\hat{\psi}_{\mathrm{I}}\left(\hat{\Gamma}^{\mathrm{I}}\right)$. We may then write for the net surface free-energy density of the pore walls

$$
\psi=\psi_{\mathrm{S}}+L \hat{\psi}_{\mathrm{I}}=\psi_{\mathrm{S}}\left(\Gamma_{\mathrm{S}}, e\right)+(1+e) \hat{\psi}_{\mathrm{I}}\left(\hat{\Gamma}_{\mathrm{I}}\right)
$$

Experiments of pore filling probe the surface stress on the conditions of controlled (through the fixed vapor pressure) chemical potential of the adsorbate. When exploring the consequences of Eq. (22) for $f$, we therefore need to consider variations of free energy with $e$ at constant $\mu$. This requires discussing $\gamma$, which is the appropriate Legendre transform of $\psi$. We write the transform as

$$
\begin{aligned}
\gamma(\mu, e) & =\psi(\Gamma, e)-\mu \Gamma \\
& =\psi_{\mathrm{S}}\left(\Gamma_{\mathrm{S}}, e\right)-\mu \Gamma_{\mathrm{S}}+(1+e)\left(\hat{\psi}_{\mathrm{I}}\left(\hat{\Gamma}_{\mathrm{I}}\right)-\mu \hat{\Gamma}_{\mathrm{I}}\right) \\
& =\gamma_{\mathrm{S}}(\mu, e)+(1+e) \hat{\gamma}_{\mathrm{I}}(\mu) .
\end{aligned}
$$

Taking the strain derivative at constant chemical potential, accounting for the fact that $f$ is only interesting at essentially zero strain, and identifying the derivative of the first term with $f_{0}$ from the equations above results in

$$
f^{\mathrm{BV}}=\left.\frac{d \gamma}{d e}\right|_{\mu}=\left.\frac{d \gamma_{\mathrm{S}}}{d e}\right|_{\mu}+\hat{\gamma}_{\mathrm{I}}(\mu),
$$

which makes use of $\gamma=\gamma_{\mathrm{S}}+(1+e) \hat{\gamma}_{\mathrm{I}}$ [Eq. (23)]. In view of the adsorption equation (13), one thus obtains

$$
f^{\mathrm{BV}}=f_{0}+\zeta \Gamma_{\mathrm{S}}(\mu)-\int_{-\infty}^{\mu} \hat{\Gamma}_{\mathrm{I}}(\tilde{\mu}) d \tilde{\mu} .
$$

Equation (25) naturally reconciles the empirical observation of an approximately linear variation of $f$ with $\Gamma$ during specific adsorption, as in the early stages of pore filling, with the prediction of Bangham's law that $f$ varies as $\hat{\gamma}$ in later stages of pore filling. Note that the adsorbate molecules on specific sites and in the incommensurate layer both contribute to changing the surface stress, yet differently and separately. Note also that $\hat{\gamma}_{\mathrm{I}}$ is only that part of the surface tension that comes from adsorption in the incommensurate layer.

Approach to saturation stage. An approximate discussion of surface stress of the solid-liquid interface $f^{\mathrm{BL}}$ may be based on the considerations of the solid-liquid surface tension $\gamma^{\mathrm{BL}}$ [see Eq. (19) in Sec. IV B]. Similarly to Eq. (25), but using the reference state at $\mu_{0}$, we have the following equation for the surface stress of the solid-liquid interface:

$$
f^{\mathrm{BL}} \approx f_{0}+\zeta \Gamma_{\mathrm{S}}^{\mathrm{max}}+\left.\Delta \psi^{\mathrm{BV}}\right|_{\mu=\mu_{0}}-\int_{\mu_{0}}^{\mu} \hat{\Gamma}_{\mathrm{I}}^{\mathrm{BL}}(\tilde{\mu}) d \tilde{\mu} .
$$

\section{F. Equilibrium conditions}

A variational approach to energy minimization can be based on Eq. (1) with the superficial free-energy density of Eq. (7), postulating for the open system (exchange of components, amount $N_{i}$, with the environment) at equilibrium that

$$
\delta \mathfrak{F}=\mu \delta N .
$$

Our problem requires the consideration of two distinct types of equilibrium conditions: First, equilibria relating to elastic deformation, and specifically elastic deformation of the solid in which no atomic bonds are broken or new bonds formed, and in which the referential area of all surfaces or interfaces remains invariant. Second, equilibria in which the referential areas of the surfaces are allowed to vary. We treat these equilibria separately and start out with the second case.

Equilibria in which the referential surface area changes: fluid. In the present context, this concerns the meniscus, where exchange of matter between fluid and gas changes the fluid volume and, thereby, its surface area. The equations of state for the chemical potential in the vapor [Eq. (15)] and in the incompressible liquid $\mu=\mu_{0}+\Omega^{\mathrm{L}} P^{\mathrm{L}}$ imply the KelvinLaplace equation [115] that relates the pressure $P^{\mathrm{L}}$ in the liquid to the vapor pressure $p$ in the gas above the meniscus through

$$
P^{\mathrm{L}}=\frac{R_{\mathrm{g}} T}{\Omega^{\mathrm{L}}} \ln \left(\frac{p}{p_{0}}\right),
$$

where $\Omega^{\mathrm{L}}$ is the molar volume in the liquid.

Equilibria in which the referential surface area changes: solid. Once the fluid has equilibrated (see above), one may take the position and the shape of the fluid meniscus as constant in the laboratory frame and consider the following variation that exclusively involves the solid: Let us apply a rigid-body displacement, by the small distance $\delta z$, of the region of solid underneath the meniscus [Fig. 4(a)]. Specifically, we take the displacement to be in the tangent plane of the solid surface, perpendicular to the triple line, and directed inward, into the fluid. The amount of wetted area of the solid surface, measured, as above, in (referential) coordinates of the solid, changes by $\delta A=m \delta z$ where $m$ is the line length of the meniscus. The variation does the work (work of spreading) $\delta W=+m \delta z\left(\gamma^{\mathrm{BV}}-\gamma^{\mathrm{BL}}\right)$. At equilibrium, this work must be compensated by work against opposing stresses. Since the fluid configuration is stationary, the fluid does not contribute. Instead, and in the absence of external traction forces on the solid, compensating stresses in the solid are required. This condition needs to be accounted for in the analysis of equilibrium.

A perfectly analogous argument considers a variation where the solid remains stationary in the laboratory frame and the meniscus moves along the atoms of the solid surface [Fig. 4(a)]. This process again changes the wetted referential area and, thereby, the net free energy. At equilibrium, pressure in the fluid is required for compensating this energy change. Thereby, the pressure in the fluid is linked to the forces acting to displace the triple line relative to the atoms of the solid surface.

The above considerations require that a line force is introduced, which acts in the plane of the interface, normal to the triple line and proportional to the line length. The associated line tension (force per length of triple line), which might be termed a "spreading tension," has the magnitude

$$
\lambda=\gamma^{\mathrm{BL}}-\gamma^{\mathrm{BV}} .
$$


(a)

(b)

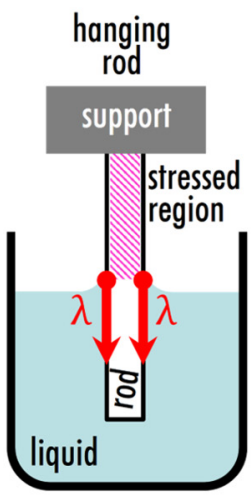

$$
\underset{\text { standing }}{\text { rod }}
$$
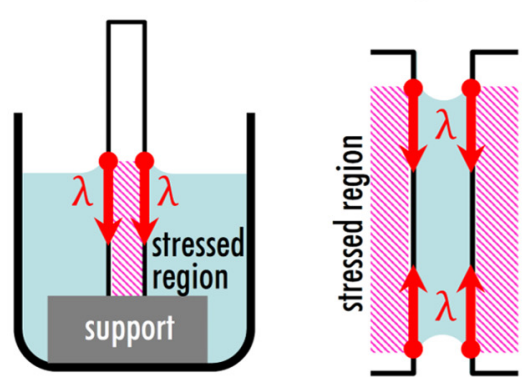

FIG. 4. Spreading of the liquid and the associated stresses. (a) Schematic representation of tangential rigid-body displacements of a section of solid (circles: atoms of the solid) relative to the meniscus. In the laboratory frame, elastic strain of the pore walls will displace the solid, pressure change in the liquid will displace the meniscus. Colored circles highlight atoms that changed their state from dry to wetted after the displacement. The change of state reduces the net surface energy, requiring compensating work terms in the solid and in the liquid. A traction force, the spreading tension $\lambda$, thus acts at the triple line, tending to pull the solid into the liquid. This action is balanced by stress in the solid and by pressure in the liquid. (b) Location of the compensating stresses in partly wetted solids of different configuration as labeled in the figure. Dry or wet regions of the solid end up stressed, depending on where the counterforces act.

In a configuration that favors wetting $\left(\gamma^{\mathrm{BV}}>\gamma^{\mathrm{BL}}\right)[3,4]$, one has $\lambda<0$ and the spreading tension tends to pull the solid into the liquid. Weijs et al. $[14,15]$ have highlighted the relevance of this force by pointing out that it is responsible for a stress in the nonwetted section of a sheet or rod that is suspended from above and partly immersed in a liquid. Figure 4(b) shows this configuration, along with variants. The figure emphasizes that the stressed regions may be underneath either, the dry or the wetted part of the solid surface, depending on where the counter forces act.

For the configuration considered below, the partially wetted circular pore of radius $r$, it is the solid underneath the wetted pore walls that experiences the stress [Fig. 4(b)]. Furthermore, with attention to the liquid, it is noted that the net force due to the spreading tension along the entire triple line is $2 \pi r \lambda$. Since that force needs to be compensated by the pressure $P^{\mathrm{L}}$ acting over the cross section $\pi r^{2}$ of the pore, one finds that the spreading tension is related to the pressure in the fluid by

$$
\lambda=-\frac{r}{2} P^{\mathrm{L}} .
$$

Purely elastic equilibria with constant surface areas. The equilibrium condition for the stress in the bulk is

$$
\operatorname{div} \mathbf{S}=\mathbf{0},
$$

whereas the local equilibrium at the surface, loaded by a fluid at pressure $P^{\mathrm{L}}$, in the absence of external load satisfies the Gurtin-Murdoch condition $[34,84,85]$

$$
\mathbf{S} \cdot \mathbf{n}+\operatorname{div}_{\mathrm{S}} \mathbb{S}+L P^{\mathrm{L}} \mathbf{n}=\mathbf{0},
$$

with $\operatorname{div}_{S}$ the surface divergence operator and $\mathbf{n}$ the local outer (as seen from the solid) surface normal. This condition specifies the normal stress component at the surface. Note that strains are small so that we may here consider $L=1$ as an excellent approximation.

The remaining stress components are constrained by the mean stress condition of Ref. [116]. Assuming once more the absence of external load, this balance equation reads as

$$
\int_{\mathrm{B}} \mathbf{S} d V+\int_{\mathrm{S}} \mathbf{S} d A=\mathbf{0} .
$$

Here, $S$ denotes the entire surface of the solid, including the pore walls and the outer surface in contact with the vapor. The impact of the outer surface is typically negligible for the problem at hand. An extended form of Eq. (33) is useful here [117]. Assume that the traction everywhere on the surface of the solid can be written as $\mathbf{T}_{0} \cdot \mathbf{n}$, with $\mathbf{T}_{0}$ a constant tensor. Then,

$$
\int_{\mathbf{B}}\left(\mathbf{S}-\mathbf{T}_{0}\right) d V+\int_{\mathbf{S}} \mathbb{S} d A=\mathbf{0} .
$$

This result will be useful when analyzing the consequences of the pressure in the fluid for the strain of the solid: The fluid pressure acts selectively on the pore walls, not on the outer surface, and for cylindrical pores this effectively introduces an anisotropy in the loading.

Local stress concentrations at the triple line. The considerations above focus on stresses and tractions that entail long-range strains and that, thereby, affect the macroscopic deformation of the porous solid. Previous studies $[14,15]$ have highlighted that there are also significant local stress and strain concentrations at the solid-liquid-vapor triple line, which are at least partly caused by jumps in surface stress across the line and by the normal component of the traction exerted by the meniscus. These local phenomena, which are not included in our analysis, may contribute significantly to the deformation of membranes with short and thick pores. Yet, it is readily seen that their impact on the macroscopic sorption strain may be neglected when attention is restricted to the limit of long narrow pores. Our discussion henceforth focuses on that limiting case.

The integral, over the ring-shaped triple line, of the normal component of the triple-line traction vanishes. Saint-Venant's principle [118] then implies that the resulting stress field must vanish at distances larger than the pore diameter. In other words, the stress field is local and its contributions to the mean 
strain are small in the limit of long narrow pores. The impact of the surface stresses on the mean stress is rigorously accounted for by Eq. (33); this implies that surface stress jumps at triple lines can have no consequence for the mean strain.

We conclude that the local stress concentrations that are demonstrated by the atomistic simulations of Ref. [14], though compatible with our considerations, are not relevant for sorption strain in long narrow pores.

\section{ARRAY OF PARALLEL CYLINDRICAL PORES WITH HIGH ASPECT RATIO}

\section{A. Geometry}

As an idealized geometry, we consider a planar sheet (membrane) of thickness $l$ with a hexagonal array of pores penetrating through the entire thickness of the sheet, and we restrict attention to pores that are much longer than they are wide. Many mesoporous materials exhibit this type of geometry: templated porous silica [119-122] and carbons $[123,124]$, porous alumina $[125,126]$ and titania $[120,127]$, and porous silicon $[50,128,129]$. The pore (inner) radius is $r$, and the pore spacing is such that the porosity (the void volume fraction) is $\phi$. This gives the specific surface area (area per volume of solid)

$$
\alpha=\frac{2}{r} \frac{\sqrt{\phi}}{1-\phi} .
$$

To good approximation we may analyze a simplified geometry, where the representative volume element (RVE) is a cylindrical tube with inner radius $r$ and with the outer radius $R$ adjusted so that $\phi$ matches the actual porous membrane $[50,66]$. This requires that

$$
R=\frac{r}{\sqrt{\phi}}
$$

\section{B. Boundary conditions}

We consider the following:

(1) The outer surfaces of the membrane can displace freely and are free of traction forces; no capillary forces act there.

(2) The pores are filled with a fluid at pressure $P^{\mathrm{L}}$; that pressure acts on the pore wall.

(3) The filling is almost complete, but a very small underfilling allows for the menisci to slide freely up and down along the pore walls when the solid is elastically deformed [130].

(4) An isotropic surface stress acts at the pore walls.

This scenario leads to the following boundary conditions (with $\mathbf{n}$ a radial unit vector):

(i) The outer surface of the RVE must be free of stress:

$$
\mathbf{S} \cdot \mathbf{n}=0 \quad \text { radial, outer surface. }
$$

(ii) Equation (32) implies here for the radial stress component in the solid right underneath the pore wall:

$$
\mathbf{n} \cdot \mathbf{S} \cdot \mathbf{n}=\frac{1}{r} f-P^{\mathrm{L}} \quad \text { radial, inner surface. }
$$

(iii) The pressure in the liquid acts on the pore walls but not on the outer surface of the porous membrane. This is embodied in the traction $\mathbf{T}_{0} \mathbf{n}$ with $\mathbf{T}_{0}=-P^{\mathrm{L}} \mathbf{Q}$, where $\mathbf{Q}$ is a unit tensor in the plane of the sheet, $\mathbf{Q}=\mathbf{e}_{x} \otimes \mathbf{e}_{y}$ with $\mathbf{e}_{x}$ and $\mathbf{e}_{y}$ orthonormal in-plane unit vectors. By using this notion with the generalized capillary equation (34), and denoting by a an axial unit vector, one would obtain a condition for the axial stress in the form $\langle\mathbf{a} \cdot \mathbf{S} \cdot \mathbf{a}\rangle(1-\phi) / \phi=-2 f / r$. Yet, this expression ignores line forces and, specifically, the spreading tension. That quantity imposes an axial stress that acts over and on top of the one due to $f$, yet that scales with the geometry in the same way. In the above axial stress condition, one may therefore replace $f$ by $f+\lambda$. When accounting for Eq. (30), one thus obtains for the axial stress balance

$$
\langle\mathbf{a} \cdot \mathbf{S} \cdot \mathbf{a}\rangle \frac{1-\phi}{\phi}=-\frac{2}{r} f+P^{\mathrm{L}} \quad \text { axial. }
$$

The most remarkable aspect of the above is that the surface tension of the meniscus, which determines the pressure in the fluid, enters the boundary conditions in a different way than the surface stress. This is apparent when comparing Eqs. (38) and (39).

\section{Displacement field and solutions}

The displacement field that satisfies Eq. (31) and that reproduces the cylindrical symmetry is here $u_{r}=c_{1} \rho+c_{2} / \rho$ in the radial direction and $u_{z}=c_{3} z$ axially, with the $c_{j}$ constants $(j=1,2,3)$ and $\rho, z$ position coordinates in radial and axial directions, respectively [66]. We compute the corresponding stress field by assuming isotropic elasticity with Young's modulus $Y$ and Poisson's ratio v. Solving for the boundary conditions (38) and (39) results in expressions for the $c_{j}$, which then allow to compute the displacements and strains in mechanical equilibrium.

\section{Relevant measures for deformation}

We consider the following measures for the deformation of the porous membrane:

(i) The out-of-plane (plane of the membrane) strain, parallel to the pores, can be measured by dilatometry or by diffraction. This strain is simply

$$
\varepsilon_{\|}=\mathbf{a} \cdot \mathbf{E} \cdot \mathbf{a} .
$$

(ii) The macroscopic in-plane strain, as measured for instance by dilatometry or by the displacement of a Bragg reflection of the pore lattice as measured in small-angle scattering, is defined as

$$
\varepsilon_{\perp}=\left.\frac{1}{R} \mathbf{u}\right|_{\rho=R} \cdot \mathbf{n} .
$$

(iii) Finally, the mean in-plane strain, as measured for instance by the displacement of an in-plane Bragg reflection of the atomic crystal lattice of a crystalline membrane, is given by

$$
\varepsilon_{\perp \text { mean }}=\langle\mathbf{n} \cdot \mathbf{E} \cdot \mathbf{n}\rangle_{\text {solid }}
$$

where the brackets denote averaging over all positions in the solid.

Consistent with our assumption of isotropy in the plane, $\mathbf{n}$ in Eqs. (41) and (42) may assume any arbitrary in-plane direction. 


\section{E. Solutions for the deformations}

The boundary conditions of Sec. VB can readily be solved for the measures of deformation of Sec. VC. Here are the solutions:

$$
\begin{gathered}
\varepsilon_{\|}=\frac{\phi}{Y(1-\phi)}\left(-\frac{2 f}{r}(1-v)+P^{\mathrm{L}}(1-2 v)\right), \\
\varepsilon_{\perp}=\frac{\phi f}{Y(1-\phi)}\left(-\frac{2 f}{r}(1-v)+P^{\mathrm{L}}(2-v)\right), \\
\varepsilon_{\perp \text { mean }}=\frac{\phi}{Y(1-\phi)}\left(-\frac{f}{r}(1-3 v)+P^{\mathrm{L}}(1-2 v)\right) .
\end{gathered}
$$

In the absence of surface stress, Eq. (44) gives the expression derived in the two-dimensional case (neglecting the Poisson's ratio effects), Eq. (3) in Ref. [50].

Equations (43) and (44) can be also compared to the strains in axial and radial directions predicted by Balzer et al. [77] using the solvation pressure approach. Note that the approach of Balzer et al. requires separate derivation before and after capillary condensation. After capillary condensation, the axial strain is given by Eqs. (15) and (13b) in Ref. [77] and the normal strain is given by Eqs. (14) and (10b) in Ref. [77]. These expressions are exactly equivalent to our Eqs. (44) and (43), respectively. Balzer's expressions for strains before capillary condensation, given by their Eqs. (15) and (13a) (axial) and (14) and (10b) (normal), differ from our results. In Sec. VI we show the comparison for the numerical results.

\section{F. Pore-load moduli}

To quantify the elastic response of a porous material to the pressure in the pores, Prass et al. introduced the notion of pore-load modulus $M$ as a ratio of the fluid pressure in the pore to the measured strain of the porous material [45]. Considering the two-dimensional model of porous material with parallel channel-like pores filled with a capillary condensate, Ref. [50] derived the analytical expression for the pore-load modulus [their Eq. (4)]:

$$
M=\frac{Y}{2\left(1-v^{2}\right)} \frac{1-\phi}{\phi} .
$$

Reference [50] considered only strain normal to the pore walls, therefore, Eq. (46) describes the normal pore-load modulus. Within the presented 3D model one can introduce two different pore-load moduli: $M_{\perp}$ and $M_{\|}$, corresponding to normal and tangential strains. Equations (44) and (43) give

$$
\begin{aligned}
& M_{\perp}=\frac{Y}{(2-v)} \frac{1-\phi}{\phi}, \\
& M_{\|}=\frac{Y}{(1-2 v)} \frac{1-\phi}{\phi} .
\end{aligned}
$$

These two pore-load moduli are noticeably different, the difference between them as a function of Poisson's ratio is shown in Fig. 5. This figure also shows the difference between the normal pore-load modulus derived in the $3 \mathrm{D}$ model and the pore-load modulus calculated for the 2D model in Ref. [50]. The relative difference $\left(M_{\perp}-M\right) / M_{\perp}$ does not exceed $7 \%$.

It is emphasized that the total deformation exhibits contributions that stem from surface stress and that are not represented by the pore-load moduli of Eqs. (47) and (48). In

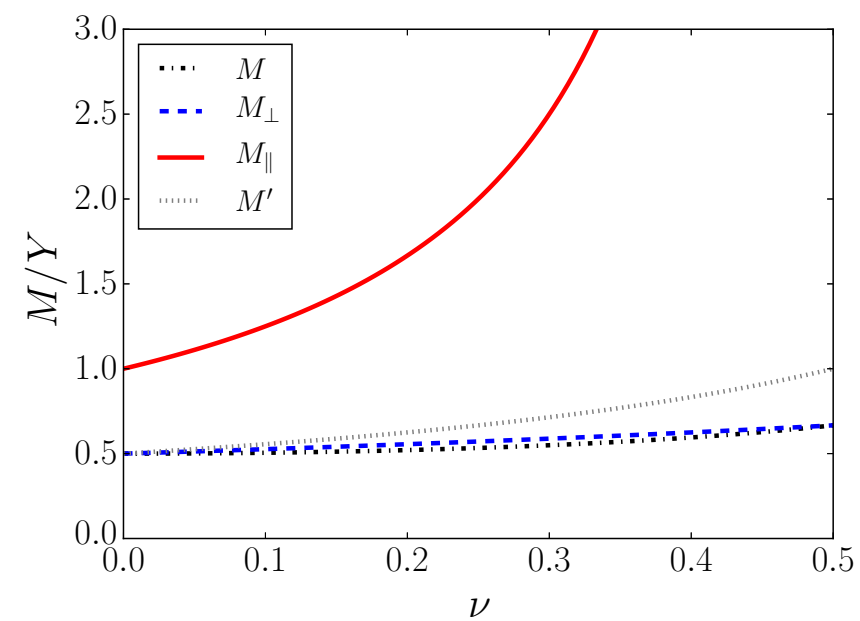

FIG. 5. Comparison of three different pore-load moduli derived in this paper: the moduli for filled pore $M_{\perp}$ corresponding to strains normal to the pore walls [Eq. (47)], $M_{\|}$along the pore walls [Eq. (48)], and the modulus $M^{\prime}$ [Eq. (49)] for the deformation in the film region. We also plot the pore-load modulus derived in Ref. [50] for the 2D model. The moduli are normalized by Young's modulus $Y$ and porosity is assumed $\phi=0.5$.

fact, the solid acts as if an (effective) pressure $P$ acted already before the onset of capillary condensation; this pressure varies with the amount of adsorbate. These extra deformations may be accounted for by (effective) pore-load moduli for the deformation of the pores due to surface stress. These terms, which are analogous to the ones introduced above, are relevant throughout the entire regime of pore filling, including the stages before capillary condensation. Contact to the above notation can be made by taking the quantity $\tilde{P}=f / r$ as an "effective pressure parameter" (not to be confused with any physical pressure). For the moduli related to the deformation normal and along the pore walls we then obtain

$$
M^{\prime} \equiv \frac{\tilde{P}}{\epsilon_{\|}}=\frac{\tilde{P}}{\epsilon_{\perp}}=\frac{Y}{2(1-v)} \frac{1-\phi}{\phi} .
$$

Figure 5 shows the difference between the pore-load modulus $M^{\prime}$ before the capillary condensation and the moduli $M_{\perp}$ and $M_{\|}$after the capillary condensation. While the difference between $M^{\prime}$ and $M_{\perp}$ is relatively small, the modulus $M_{\|}$is significantly different from the former two. The ratio $M_{\|} / M_{\perp}$ exceeds a factor of 2 and increases with Poisson's ratio. Small difference between the pore-load modulus before the capillary condensation and the modulus $M_{\perp}$ after the capillary condensation justifies the approximation of a constant poreload modulus within the whole range of vapor pressures $0 \leqslant$ $p / p_{0} \leqslant 1$ used in our previous works $[46,61,76]$.

\section{SORPTION STRAINS: NUMERICAL EXAMPLE AND COMPARISON TO SOLVATION PRESSURE APPROACH}

With an eye on illustration, we now inspect a numerical example for the strain variation during pore filling, as predicted by our Eqs. (43)-(45). We choose an example for which a detailed analysis by the theory of solvation pressure has been 
reported, namely, nitrogen adsorption on mesoporous silica [77]. This will allow us to compare the two theory approaches.

The parameters in Ref. [77] were matched to experiment. We here adopt the same parameters when applicable. Additionally, our analysis requires BET parameters which were reported in the reference. They were obtained by fitting Eq. (14) to the nitrogen adsorption data in the region $0.05 \leqslant p / p_{0} \leqslant 0.5$. The numerical values were as follows: $\phi=0.34, Y=92.0$ $\mathrm{GPa}, v=0.2, \Omega_{\mathrm{L}}=3.465 \times 10^{-5} \mathrm{~m}^{3} / \mathrm{mol}$, pore size $4.7 \mathrm{~nm}$ $(r=2.35 \mathrm{~nm})$, BET constant $C=135$, BET surface area $S_{\mathrm{BET}}=191 \mathrm{~m}^{2} / \mathrm{g}$, and monolayer capacity $\Gamma_{\mathrm{m}}=1.025 \times$ $10^{-5} \mathrm{~mol} / \mathrm{m}^{2}$ [131].

Since the adsorption coupling parameter $\zeta$ is not known for this system, we start out by ignoring specific adsorption and so treat the process as entirely incommensurate, with $\zeta=0$. This is consistent with our use of the BET adsorption isotherm. Sitespecific adsorption will be discussed at the end of the present section. All relevant equations contain a term $f_{0} / r$, and again a numerical value (here, $f_{0}$ ) is not known. Yet, the resulting strain contributions do not vary during pore filling and so may be subtracted as constants from the strain values at all $p$. We can thus ignore the $f_{0}$-related terms consistently by setting $f_{0}=0$. Finally, the change of the solid-liquid surface stress $f^{\mathrm{BL}}$ with the chemical potential for the filled pore, which is given by the last term in the right-hand side of Eq. (26), is expected much smaller than the change of the surface stress $f^{\text {BV }}$ before the capillary condensation [132]. Therefore, we neglected that change and represented $f^{\mathrm{BL}} / r$ as a horizontal line in Fig. 6(b). Figure 6 shows the results for the adsorption-induced strains, which will now be discussed.

We first consider the strains after the capillary condensation. In the example of Fig. 6, these strains are governed solely by the fluid pressure, which is given by the Kelvin-Laplace equation (28). That equation gives $P^{\mathrm{L}}=0$ as the maximum pressure in the liquid at the saturation point $\left(p=p_{0}\right)$. In the approach to saturation, $P^{\mathrm{L}}$ is negative. If a solid body were completely immersed in a fluid at $P^{\mathrm{L}}<0$, it would isotropically expand. However, the condensate here exerts the pressure only in the pores, and this leads here to compression. Note also the anisotropic strain, as embodied in our Eqs. (43) and (44). This exemplifies the difference of the pore-load moduli of Eqs. (47) and (48). The anisotropy in this example results exclusively from the interaction of the solid with the loads from the fluid and from the spreading tension; it is not related to surface stress. Note also that the mean strain varies considerably less than the macroscopic in-plane and out-of-plane strains.

Next, we discuss the strains in the regime preceding condensation. Here, we combine Eqs. (43)-(45) for the strains with Eq. (25) for the surface stress. As can be seen in Fig. 6, the mean strain variation is again small. By contrast, the macroscopic strains vary considerably.

A noteworthy feature of our example is the strain variation during the phase transformation: as condensation sets in, the in-plane as well as out-of-plane strains are both discontinuous, yet the jumps can go in different directions. Figure 6 shows the out-of-plane strain jumping to positive (expansion parallel to the pores), whereas the in-plane strain jumps to negative (contraction in the plane of the film). This prediction is a natural consequence of our model assumptions and specifically of our
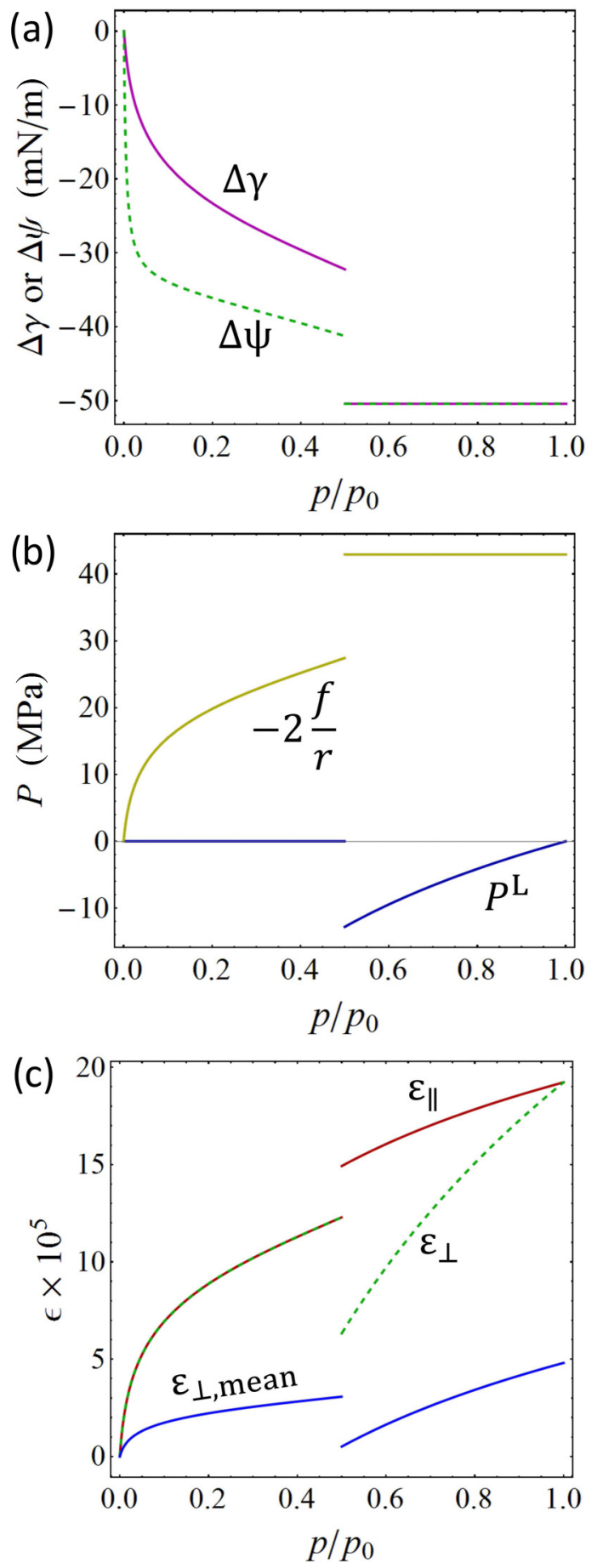

FIG. 6. Predictions of our analysis for the variation of capillary forces and strains with relative vapor pressure $p / p_{0}$ : strains from Eqs. (43), (44), and (45). Material parameters are matched to nonspecific adsorption of nitrogen on porous silica (see text). Discontinuities in graphs indicate capillary condensation, here by assumption at $p / p_{0}=0.5$. (a) Variations $\Delta \gamma$ and $\Delta \psi$ in surface tension and in surface free-energy density of the bulk-vapor or bulk-liquid interface. (b) Pressure $P^{\mathrm{L}}$ in the liquid and the effective pressure term $P^{\text {eff }}=$ $-2 f / r$. Note that $P^{\mathrm{L}}$ is governed by the liquid-vapor surface tension at the meniscus whereas $P^{\text {eff }}$ is governed by the surface stress at the solid-fluid interface. (c) The macroscopic strains $\varepsilon_{\|}$(parallel to the pore axis) and $\varepsilon_{\perp}$ (normal to the pore axis), and the volume-average strain $\varepsilon_{\perp \text { mean }}$ (also normal to the pore axis). 


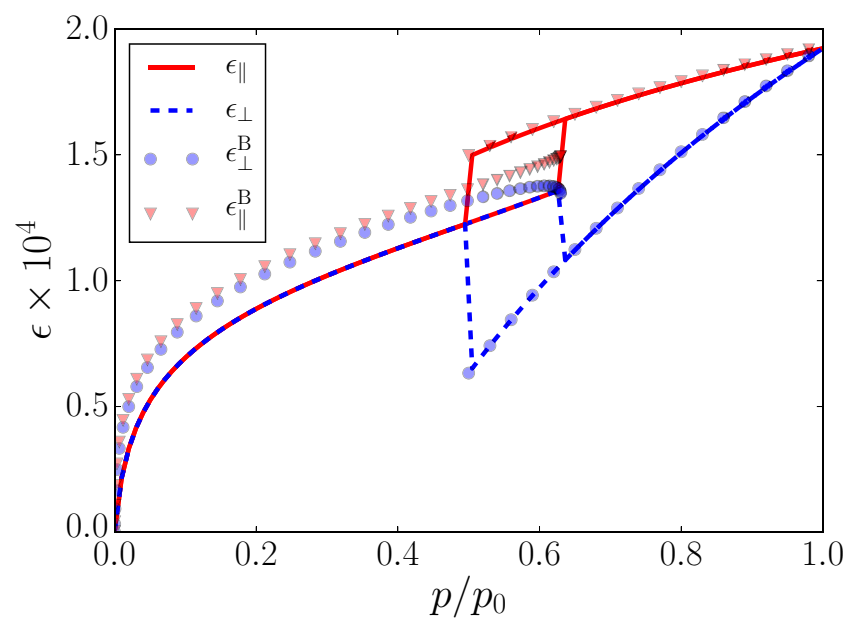

FIG. 7. Sorption strains versus reduced vapor pressure $p / p_{0}$, comparing predictions based on surface stress to those based on solvation pressure. Lines: surface-stress-based analysis of this work; results for out-of-plane (parallel to the pore axis $\epsilon_{\|}$) and in-plane $\left(\epsilon_{\perp}\right)$ strains [Eqs. (43) and (44)], as indicated by labels. Symbols: analogous results based on solvation pressure approach [77]. Parameters of both data sets are matched to nitrogen adsorption at $77 \mathrm{~K}$ in mesoporous silica.

discussion of Bangham-type behavior of the surface stress; that discussion leads to Eqs. (25) and (26).

It is of interest to compare our results to those obtained from the analysis based on the concept of solvation pressure. To this end, Fig. 7 shows the present sorption strain data (based on the concept of surface stress) along with the results of calculations from Ref. [77]. First, the two approaches provide qualitatively similar results in the adsorption regime. Second, the results for the regime of partial pore filling agree precisely. Third, both approaches predict substantial strain discontinuities, of comparable magnitude, during capillary condensation or evaporation. The above-mentioned agreement implies that the analysis of the mechanics of the solid and the two approaches are fully consistent. This is in particular clear in the regime of partial pore filling, where the loading is by the pressure in the fluid, which is simply predicted by the Kelvin-Laplace equation and so takes identical values in both approaches. Note that our approach does not predict the vapor pressure of capillary condensation or capillary evaporation. We took those points from the experimental data: for condensation $p_{c}=0.63$ and for evaporation $p_{e}=0.5$ [77].

With respect to the adsorption regime, at vapor pressures below condensation, the two approaches differ quantitatively, yet there are no apparent qualitative differences. In view of the considerable uncertainty in comparing theory to experiment, we propose that each of the two approaches is equally compatible with the available experimental observations. Note that we do not attempt to compare the predictions of our approach directly to the experimental strain data of Ref. [77] (which are reproduced in Fig. 2 above). As pointed out by those authors, their strain data represent a weighted sum of $\epsilon_{\|}$and $\epsilon_{\perp}$. Since the relative contributions of the two strains are not known, a direct comparison of the experiment to any one of the two strains of our theory is not meaningful.
Recall that the present approach bases the sorption strain in that regime on its analysis of the surface-stress variation during adsorption of an incommensurate phase, using the BET isotherm as a conservative approximation to the physics of adsorption. The adsorbate is treated as a classic hydrostatic fluid, excluding the anisotropic stress states that underlie the solvation pressure approach. Apparently, such stress states are not necessary for explaining the experimental observations. By contrast, it requires the explicit consideration of the effective forces associated with the migration of the triple line along the solid surface, and of the associated stresses in the solid. This led to our concept of a spreading tension at the line. The spreading tension again depends only on classic properties of the solid-fluid interface, namely, on the difference between the surface tensions.

In summary, the agreement between the two separate approaches in Fig. 7 demonstrates that the strains $\epsilon_{\|}$and $\epsilon_{\perp}$ can be obtained simply by combining the adsorption isotherm with the notions of surface stress and of spreading tension, without involving the concepts of disjoining or solvation pressures.

How will site-specific adsorption affect the observations? As discussed above, this question is most relevant in the early stages of adsorption. Here, since the pores are still dry, the strains of Eqs. (43)-(45) are governed by $f$ as the capillary parameter. Specifically, we consider the limit $p / p_{0} \rightarrow$ 0 , retaining the assumption of a BET adsorption isotherm [Eq. (14)]. The same result is obtained with a Langmuir isotherm. The surface-stress variation of Eq. (25) here takes the forms

$$
\begin{gathered}
f=f_{0}+\frac{p}{p_{0}} C \Gamma_{\mathrm{m}} \zeta \text { adsorption coupling, } \\
f=f_{0}-\frac{p}{p_{0}} C \Gamma_{\mathrm{m}} R_{\mathrm{g}} T \text { incommensurate }
\end{gathered}
$$

for site-specific and incommensurate adsorption, respectively. The magnitudes of the respective coefficients $\zeta$ or $-R_{\mathrm{g}} T$ are expected to be quite different:

(i) For Bangham-type behavior (incommensurate adsorbate layer), at the temperature ( $77 \mathrm{~K}$ ) of our example, we have $-R_{\mathrm{g}} T \approx-6.6 \mathrm{meV}$.

(ii) For site-specific adsorption with strong bonds, the value of $\zeta$ is in the order of $\pm 1 \mathrm{eV}, 100$-fold larger (see Sec. IVE above). Furthermore, and contrary to $R_{\mathrm{g}} T$, the adsorption coupling parameter $\zeta$ can take either sign.

(iii) Even van der Waals bonded adsorbates can have a comparatively large $\zeta$ : electronic structure density functional theory values suggest $\zeta=-64$ and $-69 \mathrm{mV}$, respectively, for $\mathrm{CH}_{4}$ and $\mathrm{CO}_{2}$ on graphene [133]. Here again, the adsorption coupling brings a much (here, tenfold) larger initial slope of strain versus partial pressure than Bangham-type behavior.

Since the surface materials parameters $C$ and $\Gamma_{\mathrm{m}}$ of Eqs. (50) and (51) can be measured independently from sorption isotherms, measurements of the deformation of porous solids can be analyzed to yield the adsorption coupling parameter $\zeta$. Aside from the quantitative difference, namely initially weaker coupling for Bangham-type surface-stress variation, there are also qualitative signatures that afford a discrimination between the two types of coupling: the explicit linear temperature dependence of the Bangham-type behavior 
of Eq. (51) is missing in the adsorption-coupling behavior of Eq. (50). Temperature-dependent studies may thus be particularly suitable for separating the contributions of the respective phenomena.

\section{DISCUSSION AND CONCLUSIONS}

We have presented a theory of adsorption-induced deformation of porous materials with parallel channel-like pores of high aspect ratio (length/width). The theory accounts explicitly for the pressure in the fluid, for the surface stress at the fluid-solid interface, and for line forces acting at the solid-liquid vapor interface. The strains along and normal to the pore axis differ for the present geometry. Our closedform stress-strain relations are based on a combination of phenomenological thermodynamics and surface mechanics. Electrosorption phenomena and actuation or sensing studies with nanoporous metals provide instances where this approach is successful and established. Our study does not aim to predict the condensation point or the adsorption isotherm. Yet, given a specific adsorption isotherm, we aim to analyze the sorption strains, exploring their directionality and establishing consistency with a classical phenomenological description.

Many previous studies of sorption strain invoke a "pressure tensor" which acts in an extended region of the fluid near the interface. By contrast, our approach considers the interface of an elastic solid with a classic fluid that supports no shear stress and that is, thus, at hydrostatic pressure. All nonhydrostatic effects at the interface, which is represented as a 2D manifold in our approach, are accounted for by its surface stress. We show that the phenomenology of sorption strain is indeed reproduced in this more conventional picture. In fact, our stress-strain relations predict the adsorption-induced deformation in the whole region of vapor pressures from $p=0$ (dry solid surface) to $p=p_{0}$ (filled pores at saturation).

A crucial element of our approach is the analysis of the variation of the surface stress with the vapor pressure. We decompose the adsorption into site-specific (commensurate) and nonspecific (incommensurate) parts. We naturally find that the incommensurate part behaves according to Bangham's law: its contribution to the change in surface stress equals its contribution to the change in surface tension (excess energy per area). One of us had previously pointed out that Bangham's law is indeed well justified for non-site-specific adsorption [76], e.g., nitrogen adsorption on amorphous silica. Yet, our analysis also shows that total change in surface stress contains a second part, which relates to specific adsorption. This part is entirely independent of the variation of the surface tension with chemical potential. Experiments, most recently in the context of actuation with nanoporous solids, demonstrate that this part can be large and that there are many experimental situations where it is the dominant part of the surface-stress variation.

We discuss a numerical example for isotherms of strain as the function of relative vapor pressure. We list three measures of strains: macroscopic strain in the directions parallel and normal to the pore walls, and average strain in the latter direction. These strains can be different. It is emphasized that the anisotropy of the strain emerges even though all interfacial stresses in our analysis, and specifically the surface stress, are isotropic in the plane of the interface. The directionality of the strains results exclusively from the anisotropic geometry of the microstructure, with pore channels aligned in one specific direction. Our results for the strains are consistent with observations for adsorption in mesoporous materials with channel-like pores, as they were reported both by dilatometric measurements [50,70,71] and by in situ x-ray diffraction $[45,46,51,70,134]$.

In the approach to saturation, our results also suggest different pore-load moduli in the directions normal and parallel to the pore walls. In the direction normal to the pore walls, the difference between the strains before and after the capillary condensation does not exceed 50\% (at $v \rightarrow 0.5$ ), and for silica $(v=0.17)$ it is only about $10 \%$. This justifies the approximation of a constant pore-load modulus within the whole range of vapor pressures used in the previous work by one of the present authors $[46,61,76]$. For the strain along the pores, the difference is noticeably higher: at $v=0$ it is a factor of 2 and for $v=0.4$ it is already a factor of 6 .

In conclusion, our work reconciles experimental observations of anisotropic strain during adsorption and capillary condensation in porous solids with a description by phenomenological thermodynamics and surface mechanics as it is widely used in the field of materials science. With regard to real nanoporous media it will be particularly interesting to study the filling-fraction-dependent mechanical loading upon capillary condensation in terms of the adsorption/desorption hysteresis. Given the importance of the strain fields at menisci triple lines the distinct distribution of liquid between adsorption and desorption, i.e., many small liquid bridges upon adsorption, much less menisci because of a coarsening of the material will result in distinct loading situations [135,136].

\section{ACKNOWLEDGMENTS}

We acknowledge support by the German research foundation (DFG) within SFB 986 "Tailor-Made Multi-Scale Materials Systems-M ${ }^{3}$ ", projects B2 and B7, and guest scientist program. We also thank $\mathrm{C}$. Balzer for providing raw data for comparison. J.W. acknowledges the most instructive discussions with M. E. Gurtin during a period of interaction. Specifically, the generalization of the mean stress condition which is our Eq. (34) was pointed out to J.W. by Gurtin.

\section{APPENDIX: DERIVATION OF EQ. (34)}

Equation (33) of the main text gives a mean stress condition that applies to solids with surface stress in the absence of external load. Here, we extend this relation to a more general loading state. The result is given as Eq. (34) in the main text.

For the purpose of the derivation we focus on the solid phase $\mathrm{B}$ alone; the fluid in the pore space is considered exclusively inasmuch as it can apply traction forces to the pore walls. We here denote by $S$ the entire surface of the solid. For the special case of a membrane, $\mathrm{S}$ includes not only the pore walls (W in the main text) but also all the planar outer surfaces. The latter are ignored in the main text since they do not noticeably contribute to the specific problem considered there.

We admit the special loading state in which the solid, everywhere on its surface $S$, experiences a traction (load per area) $\mathbf{t}$ that can be derived from the constant tensor $\mathbf{T}_{0}$ via 
$\mathbf{t}=\mathbf{T}_{0} \cdot \mathbf{n}$. As above, $\mathbf{n}$ denotes the local outer surface normal. Here, we keep $\mathbf{T}_{0}$ general, but in Sec. VB of the main text we introduce a specific choice that reproduces both the loading of the pore walls by a pressure in the fluid during capillary condensation and at the same time the vanishing load on the outer surfaces of the membrane.

We restrict attention to linear elasticity, where the superposition principle of elasticity holds. We can thus separately consider the equilibrium of the bulk with (i) the surface stress and (ii) the load due to the pressure in the fluid. The former is described by Eq. (33), and the latter will now be derived.

We apply the principle of virtual work in order to derive a condition for mechanical equilibrium. This approach exploits the following statement: For all kinematically admissible small displacement fields $\mathbf{w}(\mathbf{x})$ around the equilibrium configuration (where $\mathbf{x}$ is a position variable), the net free energy change $\delta \mathfrak{F}$ in the solid, due to the action of the displacements against the stresses in the equilibrium state, must equal the net work $\delta W$ done against the environment:

$$
\delta \mathfrak{F}=\delta W .
$$

Since this statement holds for arbitrary $\mathbf{w}(\mathbf{x})$, we can consider specifically the virtual displacement field

$$
\mathbf{w}(\mathbf{x})=\delta \mathbf{E}_{0} \cdot \mathbf{x}
$$

with $\delta \mathbf{E}_{0}$ a constant and small but otherwise arbitrary strain tensor.

We first evaluate $\delta \mathfrak{F}$. To this end, we express the variation in net free energy $\mathfrak{F}$ of the solid phase as

$$
\delta \mathfrak{F}=\int_{\mathrm{B}} \delta \Psi^{\mathrm{B}} d V=\int_{\mathrm{B}} \mathbf{S}: \delta \mathbf{E}_{0} d V .
$$

Even though we allow for the stress at equilibrium $\mathbf{S}(\mathbf{x})$ to be nonuniform in $\mathrm{B}$, our choice of $\mathbf{w}(\mathbf{x})$ as derived from a constant strain allows to move the strain outside the integral, so that the bulk contribution to the virtual work emerges as

$$
\delta \mathfrak{F}=\delta \mathbf{E}_{0}: \int_{\mathrm{B}} \mathbf{S} d V .
$$

Let us now evaluate $\delta W$, which is simply the work done against the traction forces:

$$
\delta W=\int_{\mathrm{S}} \mathbf{t} \cdot \mathbf{w}_{\mathrm{S}} d A=\int_{\mathrm{S}}\left(\mathbf{T}_{0} \cdot \mathbf{n}\right) \cdot \mathbf{w}_{\mathrm{S}} d A,
$$

with $\mathbf{w}_{\mathrm{S}}$ the displacement on S. We transform the right-hand side of this equation by means of the tensor identity

$$
\left(\mathbf{T}_{0} \cdot \mathbf{n}\right) \cdot \mathbf{w}_{\mathrm{S}}=\mathbf{T}_{0}:\left(\mathbf{w}_{\mathrm{S}} \otimes \mathbf{n}\right)
$$

and we then substitute $\mathbf{w}$ from Eq. (A2). The symbol $\otimes$ denotes the Kronecker product. Since the constant $\mathbf{T}_{0}$ can be moved out of the integral, the combined Eqs. (A2), (A5), and (A6) lead to

$$
\delta W=\mathbf{T}_{0}: \int_{\mathrm{S}}\left(\delta \mathbf{E}_{0} \cdot \mathbf{x}_{\mathrm{S}}\right) \otimes \mathbf{n} d A
$$

with $\mathbf{x}_{\mathrm{S}}$ a position on $\mathrm{S}$. Next, we convert this equation by using a divergence law (Sec. 2.4.2 in Ref. [83]), which here takes the form

$$
\int_{\mathrm{S}}\left(\delta \mathbf{E}_{0} \cdot \mathbf{x}_{\mathrm{S}}\right) \otimes \mathbf{n} d A=\int_{\mathrm{B}} \nabla\left(\delta \mathbf{E}_{0} \cdot \mathbf{x}\right) d V .
$$

The kernel of the integral on the right-hand side is simply the constant $\delta \mathbf{E}_{0}$, which can again be moved out of the integral. This yields the desired expression for the traction work in the simple form

$$
\delta W=\mathbf{T}_{0}: \delta \mathbf{E}_{0} V_{\mathrm{B}}
$$

where $V_{\mathrm{B}}$ is the volume of $\mathrm{B}$.

We can now evaluate the equilibrium condition by equating Eqs. (A4) and (A9). Since $\delta \mathbf{E}_{0}$ is an arbitrary constant tensor this yields

$$
0=\int_{\mathrm{B}}\left(\mathbf{S}-\mathbf{T}_{0}\right) d V
$$

In other words, if a solid of arbitrary shape and arbitrary microstructure is subject to tractions $\mathbf{t}$ that, everywhere on its surface, obey $\mathbf{t}=\mathbf{T}_{0} \cdot \mathbf{n}$, then the volume average of the stress in the solid is $\mathbf{T}_{0}$.

In view of the superposition principle, the above result can be added to Eq. (33). The result is Eq. (34) of the main text. That equation generalizes the mean stress condition of Ref. [116], Eq. (33) of the main text, to a more general loading state at the outer surface.
[1] G. Y. Gor, P. Huber, and N. Bernstein, Appl. Phys. Rev. 4, 011303 (2017).

[2] P. Huber, J. Phys.: Condens. Matter 27, 103102 (2015).

[3] P. G. de Gennes, F. Brochard-Wyart, and D. Queré, Capillarity and Wetting Phenomena: Drops, Bubbles, Pearls, Waves (Springer, New York, 2004).

[4] D. Bonn, J. Eggers, J. Indekeu, J. Meunier, and E. Rolley, Rev. Mod. Phys. 81, 739 (2009).

[5] D. I. Dimitrov, A. Milchev, and K. Binder, Phys. Rev. Lett. 99, 054501 (2007).

[6] S. Gruener, T. Hofmann, D. Wallacher, A. V. Kityk, and P. Huber, Phys. Rev. E 79, 067301 (2009).

[7] Y. Xue, J. Markmann, H. Duan, J. Weissmüller, and P. Huber, Nat. Commun. 5, 4237 (2014).
[8] Z. Sadjadi, M. Jung, R. Seemann, and H. Rieger, Langmuir 31, 2600 (2015)

[9] O. Vincent, B. Marguet, and A. D. Stroock, Langmuir 33, 1655 (2017).

[10] D. I. Dimitrov, A. Milchev, and K. Binder, Phys. Chem. Chem. Phys. 10, 1867 (2008).

[11] F. Cailliez, M. Trzpit, M. Soulard, I. Demachy, A. Boutin, J. Patarin, and A. H. Fuchs, Phys. Chem. Chem. Phys. 10, 4817 (2008).

[12] C. Py, P. Reverdy, L. Doppler, J. Bico, B. Roman, and C. N. Baroud, Phys. Rev. Lett. 98, 156103 (2007).

[13] A. Marchand, S. Das, J. H. Snoeijer, and B. Andreotti, Phys. Rev. Lett. 109, 236101 (2012). 
[14] J. H. Weijs, B. Andreotti, and J. H. Snoeijer, Soft Matter 9 , 8494 (2013).

[15] B. Andreotti, O. Baumchen, F. Boulogne, K. E. Daniels, E. R. Dufresne, H. Perrin, T. Salez, J. H. Snoeijer, and R. W. Style, Soft Matter 12, 2993 (2016).

[16] S. Karpitschka, A. Pandey, L. A. Lubbers, J. H. Weijs, L. Botto, S. Das, B. Andreotti, and J. H. Snoeijer, Proc. Natl. Acad. Sci. USA 113, 7403 (2016).

[17] J. Bico, B. Roman, L. Moulin, and A. Boudaoud, Nature (London) 432, 690 (2004).

[18] C. Duprat, S. Protiere, A. Y. Beebe, and H. A. Stone, Nature (London) 482, 510 (2012).

[19] Q. Zhao, J. W. Dunlop, X. Qiu, F. Huang, Z. Zhang, J. Heyda, J. Dzubiella, M. Antonietti, and J. Yuan, Nat. Commun. 5, 4293 (2014).

[20] C. Ganser, G. Fritz-Popovski, R. Morak, P. Sharifi, B. Marmiroli, B. Sartori, H. Amenitsch, T. Griesser, C. Teichert, and O. Paris, Beilstein J. Nanotechnol. 7, 637 (2016).

[21] M. Boudot, H. Elettro, and D. Grosso, ACS Nano 10, 10031 (2016).

[22] D. Van Opdenbosch, G. Fritz-Popovski, W. Wagermaier, O. Paris, and C. Zollfrank, Adv. Mater. 28, 5235 (2016).

[23] J. Biener, A. Wittstock, L. Zepeda-Ruiz, M. Biener, V. Zielasek, D. Kramer, R. Viswanath, J. Weissmüller, M. Bäumer, and A. Hamza, Nat. Mater. 8, 47 (2009).

[24] Z. J. Pan and L. D. Connell, Int. J. Coal Geol. 85, 257 (2011).

[25] G. Chen, J. Yang, and Z. Liu, Energy Fuels 26, 4583 (2012).

[26] T. Chen, X.-T. Feng, and Z. Pan, Int. J. Coal Geol. 150, 64 (2015).

[27] R. H. Baughman, C. X. Cui, A. A. Zakhidov, Z. Iqbal, J. N. Barisci, G. M. Spinks, G. G. Wallace, A. Mazzoldi, D. De Rossi, A. G. Rinzler et al., Science 284, 1340 (1999).

[28] A. E. Aliev, J. Y. Oh, M. E. Kozlov, A. A. Kuznetsov, S. L. Fang, A. F. Fonseca, R. Ovalle, M. D. Lima, M. H. Haque, Y. N. Gartstein et al., Science 323, 1575 (2009).

[29] J. Biener, S. Dasgupta, L. H. Shao, D. Wang, M. A. Worsley, A. Wittstock, J. R. I. Lee, M. M. Biener, C. A. Orme, S. O. Kucheyev et al., Adv. Mater. 24, 5083 (2012).

[30] J. Weissmüller, R. N. Viswanath, D. Kramer, P. Zimmer, R. Würschum, and H. Gleiter, Science 300, 312 (2003).

[31] D. Kramer, R. Viswanath, and J. Weissmüller, Nano Lett. 4, 793 (2004).

[32] H. J. Jin, X. L. Wang, S. Parida, K. Wang, M. Seo, and J. Weissmüller, Nano Lett. 10, 187 (2010).

[33] C. Cheng, J. Weissmüller, and A. H. W. Ngan, Adv. Mater. 28, 5315 (2016).

[34] J. Weissmüller and D. Kramer, Langmuir 21, 4592 (2005).

[35] C. Stenner, L.-H. Shao, N. Mameka, and J. Weissmüller, Adv. Funct. Mater. 26, 5174 (2016).

[36] F. T. Meehan, Proc. R. Soc. London A 115, 199 (1927).

[37] D. H. Bangham and N. Fakhoury, Nature (London) 122, 681 (1928).

[38] D. Yates, Proc. Phys. Soc. London, Sect. B 65, 80 (1952).

[39] C. H. Amberg and R. McIntosh, Can. J. Chem. 30, 1012 (1952).

[40] B. Bering, O. Krasil'nikova, A. Sarakhov, V. Serpinskii, and M. Dubinin, Bull. Acad. Sci. USSR, Div. Chem. Sci. 26, 2258 (1977).

[41] O. Krasil'nikova, B. Bering, V. Serpinskii, and M. Dubinin, Russ. Chem. Bull. 26, 1099 (1977).
[42] C. Balzer, T. Wildhage, S. Braxmeier, G. Reichenauer, and J. P. Olivier, Langmuir 27, 2553 (2011).

[43] C. Balzer, S. Braxmeier, A. V. Neimark, and G. Reichenauer, Langmuir 31, 12512 (2015).

[44] P. Kowalczyk, C. Balzer, G. Reichenauer, A. P. Terzyk, P. A. Gauden, and A. V. Neimark, Carbon 103, 263 (2016).

[45] J. Prass, D. Müter, P. Fratzl, and O. Paris, Appl. Phys. Lett. 95, 083121 (2009).

[46] G. Y. Gor, O. Paris, J. Prass, P. A. Russo, M. M. L. Ribeiro Carrott, and A. V. Neimark, Langmuir 29, 8601 (2013).

[47] P. Sharifi, B. Marmiroli, B. Sartori, F. Cacho-Nerin, J. Keckes, H. Amenitsch, and O. Paris, Bioinspired, Biomimetic Nanobiomater. 3, 183 (2014).

[48] K. Schappert and R. Pelster, Europhys. Lett. 105, 56001 (2014).

[49] G. Dolino, D. Bellet, and C. Faivre, Phys. Rev. B 54, 17919 (1996).

[50] G. Y. Gor, L. Bertinetti, N. Bernstein, T. Hofmann, P. Fratzl, and P. Huber, Appl. Phys. Lett. 106, 261901 (2015).

[51] H. S. Cho, H. Deng, K. Miyasaka, Z. Dong, M. Cho, A. V. Neimark, J. K. Kang, O. M. Yaghi, and O. Terasaki, Nature (London) 527, 503 (2015).

[52] S. Krause, V. Bon, I. Senkovska, U. Stoeck, D. Wallacher, D. M. Többens, S. Zander, R. S. Pillai, G. Maurin, F.-X. Coudert et al., Nature (London) 532, 348 (2016).

[53] T. R. Beck, J. Phys. Chem. 73, 466 (1969).

[54] H. Ibach, Surf. Sci. Rep. 29, 195 (1997).

[55] W. Haiss, Rep. Prog. Phys. 64, 591 (2001).

[56] N. Vasiljevic, T. Trimble, N. Dimitrov, and K. Sieradzki, Langmuir 20, 6639 (2004).

[57] M. Smetanin, R. N. Viswanath, D. Kramer, D. Beckmann, T. Koch, L. A. Kibler, D. M. Kolb, and J. Weissmüller, Langmuir 24, 8561 (2008).

[58] M. C. Lafouresse, U. Bertocci, and G. R. Stafford, J. Electrochem. Soc. 160, H636 (2013).

[59] L. H. Shao, H. J. Jin, and J. Weissmüller, in Nanoporous Gold: From an Ancient Technology to a High-Tech Material, RSC Nanoscience \& Nanotechnology, Vol. 22, edited by A. Wittstock, J. Biener, J. Erlebacher, and M. Bäumer (Royal Society of Chemistry, Cambridge, UK, 2012), pp. 137-166.

[60] T. S. Jakubov and D. E. Mainwaring, Phys. Chem. Chem. Phys. 4, 5678 (2002).

[61] G. Y. Gor and A. V. Neimark, Langmuir 26, 13021 (2010).

[62] E. Ustinov and D. Do, Carbon 44, 2652 (2006).

[63] P. I. Ravikovitch and A. V. Neimark, Langmuir 22, 10864 (2006).

[64] G. W. Scherer, J. Am. Ceram. Soc. 69, 473 (1986).

[65] M. Vandamme, L. Brochard, B. Lecampion, and O. Coussy, J. Mech. Phys. Solids 58, 1489 (2010).

[66] J. Weissmüller, H. L. Duan, and D. Farkas, Acta Mater. 58, 1 (2010).

[67] G. Fraux and F.-X. Coudert, Chem. Commun. 53, 7211 (2017).

[68] L.-H. Shao, H.-J. Jin, R. N. Viswanath, and J. Weissmüller, Europhys. Lett. 89, 66001 (2010).

[69] G. Y. Gor and N. Bernstein, Langmuir 32, 5259 (2016).

[70] C. Balzer, R. Morak, M. Erko, C. Triantafillidis, N. Hüsing, G. Reichenauer, and O. Paris, Z. Phys. Chem. 229, 1189 (2015).

[71] A. Grosman, J. Puibasset, and E. Rolley, Europhys. Lett. 109, 56002 (2015). 
[72] E. Rolley, N. Garroum, and A. Grosman, Phys. Rev. B 95, 064106 (2017).

[73] J. Walton, D. Tildesley, J. Rowlinson, and J. Henderson, Mol. Phys. 48, 1357 (1983).

[74] B. Coasne, Y. Long, and K. Gubbins, Mol. Simul. 40, 721 (2014).

[75] P. B. Balbuena, D. Berry, and K. E. Gubbins, J. Phys. Chem. 97, 937 (1993).

[76] G. Y. Gor and N. Bernstein, Phys. Chem. Chem. Phys. 18, 9788 (2016).

[77] C. Balzer, A. M. Waag, S. Gehret, G. Reichenauer, R. Morak, L. Ludescher, O. Paris, F. Putz, M. Elsaesser, N. Hüsing et al., Langmuir 33, 5592 (2017).

[78] T. Horikawa, D. D. Do, and D. Nicholson, Adv. Colloid Interface Sci. 169, 40 (2011).

[79] P. A. Monson, Microporous Mesoporous Mater. 160, 47 (2012).

[80] M. Thommes and K. A. Cychosz, Adsorption 20, 233 (2014).

[81] D. Kramer and J. Weissmüller, Surf. Sci. 601, 3042 (2007).

[82] J. W. Cahn, Thermodynamics of Solid and Fluid Surfaces (ASM, Metals Park, Ohio, 1978), pp. 3-23.

[83] J. Bonet and R. D. Wood, Nonlinear Continuum Mechanics for Finite Element Analysis (Cambridge University Press, Cambridge, 2008).

[84] M. Gurtin and A. I. Murdoch, Arch. Ration. Mech. Anal. 57, 291 (1975).

[85] M. E. Gurtin, J. Weissmüller, and F. Larché, Philos. Mag. A 78, 1093 (1998).

[86] The use of Lagrangian coordinates implies that $\mathbf{E}$ is an "Euler strain" and that $\mathbf{S}$ is a "second Piola-Kirchhoff stress"; see Ref. [83] and discussion in Ref. [137].

[87] P. Müller and A. Saul, Surf. Sci. Rep. 54, 157 (2004).

[88] P. Bak, Rep. Prog. Phys. 45, 587 (1982).

[89] J. P. Rabe and S. Buchholz, Science 253, 424 (1991).

[90] B. M. Ocko, O. M. Magnussen, J. X. Wang, and T. Wandlowski, Phys. Rev. B 53, R7654(R) (1996).

[91] O. M. Magnussen, Chem. Rev. 102, 679 (2002).

[92] K. Tamura, J. X. Wang, R. R. Adzic, and B. M. Ocko, J. Phys. Chem. B 108, 1992 (2004).

[93] G. R. Stafford and U. Bertocci, J. Phys. Chem. B 110, 15493 (2006).

[94] E. A. Ustinov, J. Chem. Phys. 142, 074701 (2015).

[95] A. J. Bard and L. R. Faulkner, Electrochemical Methods, Fundamentals and Applications (Wiley, New York, 1980).

[96] P. Huber and K. Knorr, Phys. Rev. B 60, 12657 (1999).

[97] P. Huber, D. Wallacher, and K. Knorr, Phys. Rev. B 60, 12666 (1999).

[98] A. Henschel, P. Kumar, T. Hofmann, K. Knorr, and P. Huber, Phys. Rev. E 79, 032601 (2009).

[99] D. Wallacher, R. Ackermann, P. Huber, M. Enderle, and K. Knorr, Phys. Rev. B 64, 184203 (2001).

[100] S. Gruener and P. Huber, Phys. Rev. Lett. 103, 174501 (2009).

[101] A. V. Kityk, K. Knorr, and P. Huber, Phys. Rev. B 80, 035421 (2009).

[102] J. Martin, J. Maiz, J. Sacristan, and C. Mijangos, Polymer 53, 1149 (2012).

[103] J. N. Israelachvili and R. M. Pashley, Nature (London) 306, 249 (1983).

[104] A. Hodgson and S. Haq, Surf. Sci. Rep. 64, 381 (2009).

[105] The variation in $\gamma$ during adsorption in a porous solid includes terms $f$ de. Equation (13) neglects these terms. This is justified inasmuch as the strain in capillary condensation scenarios is in the order of $10^{-4}$ or less, while surface stress is the order of $1 \mathrm{~N} / \mathrm{m}$. The associated $\Delta \gamma$ is then in the order of $f e \approx 0.1 \mathrm{mN} / \mathrm{m}$, considerably smaller than the direct effect of adsorption. Compare strain values in our Fig. 6 and a previous argument in the same sense in Sec. 3 of Ref. [138].

[106] S. Brunauer, P. H. Emmett, and E. Teller, J. Am. Chem. Soc. 60, 309 (1938).

[107] R. N. Viswanath and J. Weissmüller, Acta Mater. 61, 6301 (2013).

[108] Q. Deng and J. Weissmüller, Langmuir 30, 10522 (2014).

[109] F. Weigend, J. Weissmüller, and F. Evers, Small 2, 1497 (2006).

[110] J. Weissmüller, Electrocapillarity of Solids and its Impact on Heterogeneous Catalysis, Advances in Electrochemical Science and Engineering (Wiley-VCH, Weinheim, Germany, 2013), pp. 163-220.

[111] J. M. Albina, C. Elsässer, J. Weissmüller, P. Gumbsch, and Y. Umeno, Phys. Rev. B 85, 125118 (2012).

[112] G. R. Stafford and U. Bertocci, J. Phys. Chem. C 113, 261 (2009).

[113] Q. Deng, V. Gopal, and J. Weissmüller, Angew. Chem. 127, 13173 (2015).

[114] T. Bligaard, J. Nørskov, S. Dahl, J. Matthiesen, C. Christensen, and J. Sehested, J. Catal. 224, 206 (2004).

[115] S. J. Gregg and K. S. W. Sing, Adsorption, Surface Area and Porosity (Academic, London, 1982).

[116] J. Weissmüller and J. W. Cahn, Acta Mater. 45, 1899 (1997).

[117] The extended form of Eq. (33) was pointed out to one of the authors (J. W.) in 1997 by M. E. Gurtin. A derivation is shown in the Appendix.

[118] R. A. Toupin, Arch. Ration. Mech. Anal. 18, 83 (1965).

[119] D. Zhao, J. Feng, Q. Huo, N. Melosh, G. H. Fredrickson, B. F. Chmelka, and G. D. Stucky, Science 279, 548 (1998).

[120] P. C. A. Alberius, K. L. Frindell, R. C. Hayward, E. J. Kramer, G. D. Stucky, and B. F. Chmelka, Chem. Mater. 14, 3284 (2002).

[121] F. Hoffmann, M. Cornelius, J. Morell, and M. Fröba, Angew. Chem., Int. Ed. 45, 3216 (2006).

[122] C. Kuster, B. Reinhardt, M. Fröba, and D. Enke, Z. Anorg. Allg. Chem. 640, 565 (2014).

[123] J. K. Holt, H. G. Park, Y. M. Wang, M. Stadermann, A. B. Artyukhin, C. P. Grigoropoulos, A. Noy, and O. Bakajin, Science 312, 1034 (2006).

[124] V. Presser, M. Heon, and Y. Gogotsi, Adv. Funct. Mater. 21, 810 (2011).

[125] H. Masuda and K. Fukuda, Science 268, 1466 (1995).

[126] M. Steinhart, J. H. Wendorff, A. Greiner, R. B. Wehrspohn, K. Nielsch, J. Schilling, J. Choi, and U. Gösele, Science 296, 1997 (2002).

[127] J. M. Macak, H. Tsuchiya, L. Taveira, S. Aldabergerova, and P. Schmuki, Angew. Chem., Int. Ed. 44, 7463 (2005).

[128] L. T. Canham, Appl. Phys. Lett. 57, 1046 (1990).

[129] V. Lehmann and U. Gösele, Appl. Phys. Lett. 58, 856 (1991).

[130] We have verified that similar conclusions would be obtained if $\mathrm{M}$ were taken as pinned at the edge where $\mathrm{W}$ meets the outer surface of the membrane. 
[131] Our BET surface area slightly differs from the value $203.0 \mathrm{~m}^{2} / \mathrm{g}$ reported in Ref. [77], since we had to use a wider pressure range for fitting the BET parameters.

[132] Values of $\hat{\Gamma}_{\mathrm{I}}^{\mathrm{BL}}$ at the interface of liquid nitrogen with silica, for use with Eq. (26), are not known. We have inspected the density data, based on grand canonical Monte Carlo simulation for another simple fluid, argon, in cylindrical silica pores, in which the fluid-fluid and solid-fluid interactions are represented by the Lennard-Jones potential [139]. The decrease in the mean density of the liquid with diminishing pore radius suggests a negative superficial excess of matter, about $\hat{\Gamma}_{\mathrm{I}}^{\mathrm{BL}}=-1.7 \times$ $10^{-6} \mathrm{~mol} / \mathrm{m}^{2}$ or -0.12 dense-packed atomic monolayers. In view of Eq. (26), and applied to the numerical example of Fig. 6, this would imply a decrease in $f$ by $0.75 \mathrm{~mJ} / \mathrm{m}^{2}$ between capillary condensation and saturation. That variation is small compared to the variation of the capillary terms of Fig. 6(a) and so the effect may be neglected in our example.

[133] The estimates of $\zeta$ on graphene are based on data for adsorption enthalpy versus strain in Ref. [140] along with a thermody- namic Maxwell relation that links that data to $\varsigma$ (see Ref. [141]) and, hence, to $\zeta$.

[134] G. Günther, J. Prass, O. Paris, and M. Schoen, Phys. Rev. Lett. 101, 086104 (2008).

[135] J. H. Page, J. Liu, B. Abeles, H. W. Deckman, and D. A. Weitz, Phys. Rev. Lett. 71, 1216 (1993).

[136] V. P. Soprunyuk, D. Wallacher, P. Huber, K. Knorr, and A. V. Kityk, Phys. Rev. B 67, 144105 (2003).

[137] B. A. M. Elsner, S. Müller, S. Bargmann, and J. Weissmüller, Acta Mater. 124, 468 (2017).

[138] J. Lipkowski, W. Schmickler, D. M. Kolb, and R. Parsons, J. Electroanal. Chem. 452, 193 (1998).

[139] C. D. Dobrzanski, M. A. Maximov, and G. Y. Gor, J. Chem. Phys. 148, 054503 (2018).

[140] D. Dutta, B. C. Wood, S. Y. Bhide, K. G. Ayappa, and S. Narasimhan, J. Phys. Chem. C 118, 7741 (2014).

[141] J. Weissmüller, Nat. Catal. 1, 238 (2018). 